\title{
Asymptotics for the Ostrovsky-Hunter Equation in the Critical Case
}

\author{
Fernando Bernal-Vílchis, ${ }^{1}$ Nakao Hayashi, ${ }^{2}$ and Pavel I. Naumkin ${ }^{3}$ \\ ${ }^{1}$ Instituto de Matemáticas, UNAM, Campus Morelia, AP 61-3 Xangari, 58089 Morelia, MICH, Mexico \\ ${ }^{2}$ Department of Mathematics, Graduate School of Science, Osaka University, Osaka, Toyonaka 560-0043, Japan \\ ${ }^{3}$ Centro de Ciencias Matemáticas, UNAM, Campus Morelia, AP 61-3 Xangari, 58089 Morelia, MICH, Mexico \\ Correspondence should be addressed to Nakao Hayashi; nhayashi@math.sci.osaka-u.ac.jp
}

Received 19 July 2016; Accepted 30 August 2016; Published 23 January 2017

Academic Editor: Baoxiang Wang

Copyright (c) 2017 Fernando Bernal-Vílchis et al. This is an open access article distributed under the Creative Commons Attribution License, which permits unrestricted use, distribution, and reproduction in any medium, provided the original work is properly cited.

We consider the Cauchy problem for the Ostrovsky-Hunter equation $\partial_{x}\left(\partial_{t} u-(b / 3) \partial_{x}^{3} u-\partial_{x} \mathscr{K} u^{3}\right)=a u,(t, x) \in \mathbb{R}^{2}, u(0, x)=u_{0}(x)$, $x \in \mathbb{R}$, where $a b>0$. Define $\xi_{0}=(27 a / b)^{1 / 4}$. Suppose that $\mathscr{K}$ is a pseudodifferential operator with a symbol $\widehat{K}(\xi)$ such that $\widehat{K}\left( \pm \xi_{0}\right)=0, \operatorname{Im} \widehat{K}(\xi)=0$, and $|\widehat{K}(\xi)| \leq C$. For example, we can take $\widehat{K}(\xi)=\left(\xi^{2}-\xi_{0}^{2}\right) /\left(\xi^{2}+1\right)$. We prove the global in time existence and the large time asymptotic behavior of solutions.

\section{Introduction}

We consider the Cauchy problem for the generalized Ostrovsky-Hunter equation

$$
\begin{aligned}
\partial_{x}\left(\partial_{t} u-\frac{b}{3} \partial_{x}^{3} u-\partial_{x} f(u)\right) & =a u, \quad(t, x) \in \mathbb{R}^{2}, \\
u(0, x) & =u_{0}(x), \quad x \in \mathbb{R},
\end{aligned}
$$

where $a b>0, f(u)=\mathscr{K} u^{3}$. We assume that $\mathscr{K}$ is a pseudodifferential operator with a symbol $\widehat{K}(\xi)$ such that $\widehat{K}\left( \pm \xi_{0}\right)=0$ with $\xi_{0}=(27 a / b)^{1 / 4}$. Also we suppose that $\operatorname{Im} \widehat{K}(\xi)=0$ and $|\widehat{K}(\xi)| \leq C$. For example, we can choose $\widehat{K}(\xi)=\left(\xi^{2}-\right.$ $\left.\xi_{0}^{2}\right) /\left(\xi^{2}+1\right)$. Denote by $\Lambda(\xi)=a / \xi+(b / 3) \xi^{3}$ the symbol of the linear part of (1). The constant $\xi_{0}=(27 a / b)^{1 / 4}$ is a positive root of $\Omega(\xi)=\Lambda(\xi)-3 \Lambda(\xi / 3)=(8 b / 27) \xi^{-1}\left(\xi^{4}-27 a / b\right)=$ 0 . Our strategy of the proof of the main result is similar to the one used in [1]. We translate (1) into the ordinary differential equation by using the evolution operator related to the linear problem; then we divide the nonlinear term into resonance and nonresonance parts. Nonresonance part has an oscillating term $e^{i t \Omega(\xi)}$ which yields better time decay through the integration by parts; however the factor $1 / \Omega(\xi)$ gives us a singularity at $\xi_{0}$; see (37) for details. This is the reason why we assume the additional condition $\widehat{K}\left( \pm \xi_{0}\right)=0$ on the symbol $\widehat{K}(\xi)$.

We define the evolution group $\mathscr{U}(t)=\mathscr{F}^{-1} E \mathscr{F}$, where the multiplication factor $E=e^{-i t \Lambda(\xi)}, \Lambda(\xi)=a / \xi+(b / 3) \xi^{3}$. It is well known that the operator $\mathscr{J}=\mathscr{U}(t) x \mathscr{U}(-t)$ is a useful tool for obtaining the $\mathbf{L}^{\infty}$-time decay estimates of solutions and has been used widely for studying the asymptotic behavior of solutions to various nonlinear dispersive equations. We have

$$
\begin{aligned}
\mathscr{J} & =\mathscr{U}(t) x \mathscr{U}(-t)=\mathscr{F}^{-1} e^{-i t \Lambda(\xi)} i \partial_{\xi} e^{i t \Lambda(\xi)} \mathscr{F} \\
& =\mathscr{F}^{-1}\left(i \partial_{\xi}-t \Lambda^{\prime}(\xi)\right) \mathscr{F}=x-t \Lambda^{\prime}\left(-i \partial_{x}\right) \\
& =x-t a \partial_{x}^{-2}+t b \partial_{x}^{2},
\end{aligned}
$$

where $\Lambda^{\prime}\left(-i \partial_{x}\right)=a \partial_{x}^{-2}-b \partial_{x}^{2}$, and the antiderivative $\partial_{x}^{-1}$ is defined by the Fourier transform such that

$$
\widehat{\partial_{x}^{-1} \phi}(\xi)=(i \xi)^{-1} \widehat{\phi}(\xi) .
$$

Note that the commutators are true $[\mathscr{F}, \mathscr{L}]=0,\left[\partial_{x}, \mathscr{L}\right]=$ $0,\left[\mathscr{g}, \partial_{x}\right]=-1,\left[\partial_{x}^{-1}, x \partial_{x}\right]=-\partial_{x}^{-1}$, where $\mathscr{L}=\partial_{t}+$ $\Lambda\left(-i \partial_{x}\right)=\partial_{t}-a \partial_{x}^{-1}-(b / 3) \partial_{x}^{3}$. However, it seems that $\mathscr{J}$ does not work well on the nonlinear terms. In order to avoid the derivative loss, when estimating the norm $\left\|\partial_{x} \mathscr{F} u\right\|_{L^{2}}$ instead 
of the operators $\mathscr{J}$ we apply the modified dilation operator defined by

$$
\mathscr{P}=t \partial_{t}+\frac{1}{3} x \partial_{x}-\frac{4}{3} a \partial_{a} .
$$

Note that $\mathscr{P}$ acts well on the nonlinear terms as the firstorder differential operator and it almost commutes with $\mathscr{L}$ : $[\mathscr{P}, \mathscr{L}]=-\mathscr{L}$. Also $\mathscr{J}$ and $\mathscr{P}$ are related via the identity

$$
\mathscr{P}=t \mathscr{L}+\frac{1}{3} \mathscr{J} \partial_{x}-\frac{4}{3} a \mathscr{F},
$$

where

$$
\mathscr{I}=\partial_{a}-t \partial_{x}^{-1}
$$

Note that $[\mathscr{I}, \mathscr{L}]=0$. In order to get the estimate of $\partial_{x} \mathscr{F} u$, we will show the a priori estimates of, $t \mathscr{L} u$, and $\mathscr{I} u$. Different point compared to the previous works is to consider the estimate of $\mathscr{I} u$ since $\mathscr{I} u$ contains the term $t \partial_{x}^{-1}$ with an additional explicit time growth.

When $f(u)=u^{2}$, then (1) was introduced in [2] for modelling the small-amplitude long waves in a rotating fluid of finite depth. Therefore (1) with $f(u)=u^{2}$ is called the Ostrovsky equation. It was studied by many authors (see, e.g., [3-5] and references cited therein). When $b=0$, (1) is called the reduced Ostrovsky equation. Equation (1) has some conservation quantities, when $f(u)=\lambda|u|^{p-1} u, \lambda \in \mathbb{R}$. One of them is the zero mass conservation law which is obtained by integrating in space

$$
a \int u(t, x) d x=0
$$

under the restriction $\int u_{0}(x) d x=0$. Rewrite (1) as

$$
\partial_{t} u-\frac{b}{3} \partial_{x}^{3} u-\partial_{x} f(u)=a D_{x}^{-1} u .
$$

Multiplying both sides of (8) by $u$, integrating in space, using (7), we obtain

$$
\frac{d}{d t} \int|u(t, x)|^{2} d x+\frac{2 \lambda}{p+1} \int|u(t, x)|^{p+1} d x=0
$$

which is the conservation of the momentum. The same approach as in deriving (9) will be used for the high frequency part in order to avoid the derivative loss, when proving the existence of solutions of (1).

Local well-posedness for the Ostrovsky equation was shown in [5] in the case of the initial data

$$
u_{0} \in \mathbf{H}^{s} \cap \dot{\mathbf{H}}^{-1}, \quad s>\frac{3}{2}
$$

by using the parabolic regularization technique and limiting arguments. Their method works also for the case of the generalized nonlinearity $f(u)=|u|^{p-1} u$ and also generalized reduced Ostrovsky equation (1), since the dispersive effects were not used in the proof. Thanks to the high frequency part $u_{x x x}$, the solutions to the linear equation $\left(u_{t}-\beta u_{x x x}\right)_{x}=$ $\gamma u$ obtain a smoothing property. By using this property, in
[3], the local well-posedness for the Ostrovsky equation was shown under the condition

$$
u_{0} \in \mathbf{H}^{s} \cap \dot{\mathbf{H}}^{-1}, \quad s>\frac{3}{4} .
$$

The method of [3] depends on the linear part of the equation and also works for the nonlinearities of a general order. In $[4,6-8]$ the local well-posedness for the Ostrovsky equation was treated by the Fourier restriction norm method of [9] and in [4] the $\mathbf{H}^{-3 / 4+}$ local well-posedness was shown. We note here that the Sobolev space $\mathbf{H}^{-3 / 4}$ is considered as critical regularity concerning the Korteweg-de Vries equation.

Global well-posedness in the energy class was obtained for the Ostrovsky equation in [3] through the energy conservation law, when the initial data

$$
u_{0} \in \mathbf{H}^{1} \cap \dot{\mathbf{H}}^{-1},
$$

and $a b>0$. After their work, the global well-posedness in

$$
\mathbf{L}^{2} \cap \dot{\mathbf{H}}^{-s}, \quad 0 \leq s \leq 1,
$$

was proved in $[4,6]$ due to the $\mathbf{L}^{2}$-conservation law. The global well-posedness, in the negative order Sobolev space $\mathbf{H}^{-3 / 10+}$, was shown in [8] by using the $I$ method of [10].

We now turn to the case of the reduced Ostrovsky equation. The local well-posedness was shown in the space $\mathbf{H}^{2}$ in paper [11] and after that in $\mathbf{H}^{3 / 2+}$ in [12]. Their methods work also in the case of the general nonlinear dispersive equations with different nonlinearities. We also refer to [13, 14] for the local well-posedness in the class

$$
u_{0} \in \mathbf{H}^{m} \cap \dot{\mathbf{H}}^{-1} \quad m \geq 2 .
$$

However there are few works on the global well-posedness for the reduced Ostrovsky equation due to the lack of the smoothing property. The global well-posedness for reduced Ostrovsky equation (1) with $b=0$ and cubic nonlinearity $f(u)=u^{3}$ (which is called the short pulse equation) was obtained in [15], when the initial data

$$
\left\|\partial_{x} u_{0}\right\|_{\mathbf{H}^{1}}<1, \quad u_{0} \in \mathbf{H}^{2},
$$

whereas for the quadratic nonlinearity $f(u)=u^{2}$ (which is called the reduced Ostrovsky equation or the OstrovskyHunter equation; see $[16,17])$, it was shown in [18] when the initial data

$$
\left(1-3 \partial_{x}^{2}\right) u_{0}(x)<0, \quad u_{0} \in \mathbf{H}^{3},
$$

for all $x \in \mathbf{R}$. The time decay properties of solutions to the corresponding linear problem can be studied if we assume that the initial data decay rapidly at infinity. So the global existence was shown in [12], for the nonlinearity $f(u)=u^{p}$ with an integer $p \geq 4$, when the initial data are small and sufficiently regular:

$$
u_{0} \in \mathbf{H}^{5} \cap \mathbf{H}_{1}^{3} .
$$


In $[1,19,20]$, we considered the large time asymptotics for reduced Ostrovsky equation (1) with $b=0$ and some conditions on the order of nonlinearity.

To state our results precisely we introduce Notation and Function Spaces. We denote the Lebesgue space by $\mathbf{L}^{p}=\{\phi \in$ $\left.\mathbf{S}^{\prime} ;\|\phi\|_{\mathbf{L}^{p}}<\infty\right\}$, where the norm $\|\phi\|_{\mathbf{L}^{p}}=\left(\int|\phi(x)|^{p} d x\right)^{1 / p}$ for $1 \leq p<\infty$ and $\|\phi\|_{L^{\infty}}=\operatorname{ess.sup}_{x \in \mathbf{R}}|\phi(x)|$ for $p=\infty$. The weighted Sobolev space is

$$
\mathbf{H}_{p}^{k, s}=\left\{\varphi \in \mathbf{S}^{\prime} ;\|\phi\|_{\mathbf{H}_{p}^{k, s}}=\left\|\langle x\rangle^{s}\left\langle i \partial_{x}\right\rangle^{k} \phi\right\|_{\mathbf{L}^{p}}<\infty\right\} .
$$

$k, s \in \mathbf{R}, 1 \leq p \leq \infty,\langle x\rangle=\sqrt{1+x^{2}}$, and $\left\langle i \partial_{x}\right\rangle=$ $\sqrt{1-\partial_{x}^{2}}$. We also use the notations $\mathbf{H}^{k, s}=\mathbf{H}_{2}^{k, s}, \mathbf{H}^{k}=\mathbf{H}^{k, 0}$ shortly, if they do not cause any confusion. Let $\mathbf{C}(\mathbf{I} ; \mathbf{B})$ be the space of continuous functions from an interval I to a Banach space $\mathbf{B}$. Different positive constants might be denoted by the same letter $C$. We define the free evolution group $\mathcal{U}(t)=$ $e^{-i t \Lambda\left(-i \partial_{x}\right)}=\mathscr{F}^{-1} E \mathscr{F}$, where the multiplication factor $E(t, \xi)=$ $e^{-i t \Lambda(\xi)}$.

We are now in a position to state our main result.

Theorem 1. Assume that the initial data $u_{0} \in \mathbf{H}^{2} \cap \mathbf{H}^{1,1}$ are real-valued with a sufficiently small norm $\left\|u_{0}\right\|_{\mathrm{H}^{2} \cap \mathrm{H}^{1,1}} \leq \varepsilon$. Then there exists a unique global solution $u \in \mathbf{C}\left([0, \infty) ; \mathbf{H}^{2}\right)$ of Cauchy problem (1) satisfying the time decay estimate

$$
\|u(t)\|_{\mathbf{L}^{\infty}} \leq C \varepsilon t^{-1 / 2}
$$

Moreover there exists a unique modified final state $W_{+} \in \mathbf{L}^{\infty}$ such that the asymptotics

$$
\begin{aligned}
& u(t)=2 \operatorname{Re}^{-1 / 2} e^{-2 i t\left(a / \eta(x / t)-(b / 3) \eta(x / t)^{3}\right)} \frac{W_{+}(\eta(x / t))}{\sqrt{\Lambda^{\prime \prime}(\eta(x / t))}} \\
& \cdot \exp \left(\frac{3 i \eta(x / t) \widehat{K}(\eta(x / t))}{\langle\eta(x / t)\rangle \Lambda^{\prime \prime}(\eta(x / t))}\left|W_{+}\left(\eta\left(\frac{x}{t}\right)\right)\right|^{2}\right. \\
& \cdot \log t)+O\left(\varepsilon t^{-1 / 2-\delta}\right)
\end{aligned}
$$

is valid for $t \rightarrow \infty$ uniformly with respect to $x \in \mathbf{R}$, where $\delta>0$ is a small constant and

$$
\eta(x)=\sqrt{\frac{1}{2 b}\left(x+\sqrt{4 a b+x^{2}}\right)} .
$$

\section{Factorization Technique}

We now introduce the factorization formulas for (1). We have for the free evolution group $\mathcal{U}(t)=\mathscr{F}^{-1} E \mathscr{F}$, where the multiplication factor $E=e^{-i t \Lambda(\xi)}, \Lambda(\xi)=a / \xi+(b / 3) \xi^{3}$. Denote the Heaviside function $\theta(\xi)=1$ for $\xi>0$ and $\theta(\xi)=0$ for $\xi \leq 0$. Then for the real-valued function $u(t)=\mathscr{U}(t) \mathscr{F}^{-1} \widehat{\varphi}$ we find

$$
\begin{aligned}
\mathcal{U}(t) \mathscr{F}^{-1} \widehat{\varphi} & =2 \operatorname{Re} \mathscr{F}^{-1} \theta E \widehat{\varphi} \\
& =2 \operatorname{Re} \frac{1}{\sqrt{2 \pi}} \int_{0}^{\infty} e^{i t((x / t) \xi-\Lambda(\xi))} \widehat{\varphi}(\xi) d \xi \\
& =2 \operatorname{Re} \mathscr{D}_{t} \frac{|t|^{1 / 2}}{\sqrt{2 \pi}} \int_{0}^{\infty} e^{i t(x \xi-\Lambda(\xi))} \widehat{\varphi}(\xi) d \xi
\end{aligned}
$$

where the dilation operator $\mathscr{D}_{t} \phi=|t|^{-1 / 2} \phi\left(x t^{-1}\right)$. Note that there is a unique stationary point in the integral $\int_{0}^{\infty} e^{i t(x \xi-\Lambda(\xi))} \phi(\xi) d \xi$, which is defined by the root $\xi=\eta(x)=$ $\sqrt{(1 / 2 b)\left(x+\sqrt{4 a b+x^{2}}\right)}>0$ of the equation $\Lambda^{\prime}(\xi)=-a / \xi^{2}+$ $b \xi^{2}=x$ for all $x \in \mathbb{R}$. Thus $\Lambda^{\prime}(\eta(x))=x$ and we introduce the so-called scaling operator

$$
\left(\mathscr{B}^{-1} \phi\right)(x)=\frac{1}{\sqrt{\Lambda^{\prime \prime}(\eta(x))}} \phi(\eta(x))
$$

and the multiplication factor

$$
M(t, \eta)=e^{i t\left(\eta \Lambda^{\prime}(\eta)-\Lambda(\eta)\right)}=e^{-2 i t\left(a / \eta-(b / 3) \eta^{3}\right)} .
$$

Note that, in the case of $b=0$, then $\eta(x)$ is defined by $\Lambda^{\prime}(\xi)=$ $-a / \xi^{2}=x$; namely, $\eta(x)=\sqrt{a /|x|}$ for $x<0$. Hence

$$
\left(\mathscr{B}^{-1} \phi\right)(x)=\frac{1}{\sqrt{3 a}}\left(\frac{a}{|x|}\right)^{3 / 4} \phi\left(\sqrt{\frac{a}{|x|}}\right),
$$

for $b=0, x<0$; see [1]. Therefore $\mathscr{B}^{-1}$ is the scaling operator if the symbol $\Lambda(\xi)$ is homogeneous.

By the definition of $\mathscr{B}^{-1}$, its inverse operator is defined by

$$
(\mathscr{B} \phi)(\eta)=\sqrt{\Lambda^{\prime \prime}(\eta)} \phi\left(\Lambda^{\prime}(\eta)\right)
$$

Then we have

$$
\begin{aligned}
\mathscr{U}(t) \mathscr{F}^{-1} \phi=2 \operatorname{Re} \mathscr{D}_{t} \frac{|t|^{1 / 2}}{\sqrt{2 \pi}} \int_{0}^{\infty} e^{i t(x \xi-\Lambda(\xi))} \phi(\xi) d \xi \\
=2 \operatorname{Re} \mathscr{D}_{t} \mathscr{B}^{-1} \mathscr{B} \frac{|t|^{1 / 2}}{\sqrt{2 \pi}} \int_{0}^{\infty} e^{i t\left(\Lambda^{\prime}(\eta(x)) \xi-\Lambda(\xi)\right)} \phi(\xi) d \xi \\
=2 \operatorname{Re} \mathscr{D}_{t} \mathscr{B}^{-1} M \sqrt{\frac{|t| \Lambda^{\prime \prime}(\eta)}{2 \pi}} \int_{0}^{\infty} e^{-i t S(\eta, \xi)} \phi(\xi) d \xi \\
=2 \operatorname{Re} \mathscr{D}_{t} \mathscr{B}^{-1} M \mathscr{V} \phi,
\end{aligned}
$$

where the phase function $S(\eta, \xi)=\Lambda(\xi)-\Lambda(\eta)-\Lambda^{\prime}(\eta)(\xi-\eta)$ and the operator

$$
\begin{aligned}
\mathscr{V} \phi & =\bar{M} \mathscr{B}^{-1} \mathscr{D}_{t}^{-1} \mathscr{F}^{-1} E \theta \phi \\
& =\sqrt{\frac{|t| \Lambda^{\prime \prime}(\eta)}{2 \pi}} \int_{0}^{\infty} e^{-i t S(\eta, \xi)} \phi(\xi) d \xi .
\end{aligned}
$$


We have $\left\|\mathscr{D}_{t}^{-1} \phi\right\|_{\mathrm{L}^{2}}=\|\phi\|_{\mathrm{L}^{2}},\left\|\mathscr{F}^{-1} \phi\right\|_{\mathrm{L}^{2}}=\|\phi\|_{\mathrm{L}^{2}}$, and $\left\|\mathscr{B}^{-1} \phi\right\|_{\mathrm{L}^{2}}=\|\phi\|_{\mathrm{L}^{2}}$. Hence

$$
\begin{aligned}
\|\mathscr{V} \phi\|_{L^{2}} & =\left\|\bar{M} \mathscr{B}^{-1} \mathscr{D}_{t}^{-1} \mathscr{F}^{-1} E \theta \phi\right\|_{L^{2}}=\|\theta \phi\|_{\mathbf{L}^{2}} \\
& \leq\|\phi\|_{\mathrm{L}^{2}} .
\end{aligned}
$$

Also we decompose the inverse operator

$$
\begin{array}{rl}
\mathscr{F} & \mathcal{U}(-t) \phi=\bar{E} \mathscr{F} \phi=\frac{1}{\sqrt{2 \pi}} \int_{\mathbb{R}} e^{-i t((x / t) \xi-\Lambda(\xi))} \phi(x) d x \\
= & \frac{|t|^{1 / 2}}{\sqrt{2 \pi}} \int_{\mathbb{R}} e^{-i t(\xi x-\Lambda(\xi))} \mathscr{D}_{t}^{-1} \phi(x) d x . \\
= & \frac{|t|^{1 / 2}}{\sqrt{2 \pi}} \int_{0}^{\infty} e^{-i t\left(\xi \Lambda^{\prime}(\eta)-\Lambda(\xi)\right)} \sqrt{\Lambda^{\prime \prime}(\eta)}\left(\mathscr{B} \mathscr{D}_{t}^{-1} \phi\right) d \eta \\
= & \frac{|t|^{1 / 2}}{\sqrt{2 \pi}} \int_{0}^{\infty} e^{i t S(\eta, \xi)} \bar{M}\left(\mathscr{B} \mathscr{D}_{t}^{-1} \phi\right) \sqrt{\Lambda^{\prime \prime}(\eta)} d \eta \\
= & \mathscr{V}^{*} \bar{M} \mathscr{B} \mathscr{D}_{t}^{-1} \phi .
\end{array}
$$

Since $x=\Lambda^{\prime}(\eta)$, then

$$
\begin{aligned}
\int_{\mathbb{R}} & e^{-i t(\xi x-\Lambda(\xi))} \mathscr{D}_{t}^{-1} \phi(x) d x \\
& =\int_{\mathbb{R}} e^{-i t\left(\xi \Lambda^{\prime}(\eta)-\Lambda(\xi)\right)} \mathscr{D}_{t}^{-1} \phi\left(\Lambda^{\prime}(\eta)\right) \Lambda^{\prime \prime}(\eta) d \eta \\
& =\int_{0}^{\infty} e^{-i t\left(\xi \Lambda^{\prime}(\eta)-\Lambda(\xi)\right)} \sqrt{\Lambda^{\prime \prime}(\eta)}\left(\mathscr{B} \mathscr{D}_{t}^{-1} \phi\right) d \eta \\
& =\int_{0}^{\infty} e^{i t 5(\eta, \xi)} \bar{M}\left(\mathscr{B} \mathscr{D}_{t}^{-1} \phi\right) \sqrt{\Lambda^{\prime \prime}(\eta)} d \eta \\
& =\frac{\sqrt{2 \pi}}{|t|^{1 / 2}} \mathscr{V}^{*} \bar{M} \mathscr{B} \mathscr{D}_{t}^{-1} \phi,
\end{aligned}
$$

Define the new dependent variable $\widehat{\varphi}=\mathscr{F} \mathscr{U}(-t) u(t)$. Since $\mathscr{F} \mathscr{U}(-t) \mathscr{L}=\partial_{t} \mathscr{F} \mathscr{U}(-t)$, where $\mathscr{L}=\partial_{t}-\partial_{x}^{-1}-(1 / 3) \partial_{x}^{3}$, applying the operator $\mathscr{F} \mathscr{U}(-t)$ to (1) we get

$$
\begin{aligned}
\partial_{t} \widehat{\varphi} & =\partial_{t} \mathscr{F} \mathscr{U}(-t) u=\mathscr{F} \mathscr{U}(-t) \mathscr{L} u \\
& =\mathscr{F} \mathscr{U}(-t) \partial_{x} \mathscr{K} u^{3}=i \xi \widehat{K}(\xi) \mathscr{F} \mathscr{U}(-t) u^{3} \\
& =i \xi \widehat{K}(\xi) \mathscr{V}^{*} \bar{M} \mathscr{B}^{-1} \mathscr{D}_{t}^{-1}\left(u^{3}\right) .
\end{aligned}
$$

Then since

$$
\begin{aligned}
\mathcal{U}(t) \mathscr{F}^{-1} \phi & =2 \operatorname{Re} \mathscr{D}_{t} \mathscr{B}^{-1} M \mathscr{V} \phi, \\
u & =\mathscr{U}(t) \mathscr{F}^{-1} \widehat{\varphi}=2 \operatorname{Re} \mathscr{D}_{t} \mathscr{B}^{-1} M \mathscr{V} \widehat{\varphi} \\
& =\mathscr{D}_{t} \mathscr{B}^{-1}(M \mathscr{V} \widehat{\varphi}+\bar{M} \overline{\mathscr{V}} \hat{\varphi}),
\end{aligned}
$$

we find the following representation:

$$
\begin{aligned}
\partial_{t} \widehat{\varphi} & =\mathscr{V}^{*} \bar{M} \mathscr{B}_{t}^{-1}\left(\partial_{x} \mathscr{K} u^{3}\right)=i \xi \widehat{K}(\xi) \\
& \cdot \mathscr{V}^{*} \bar{M} \mathscr{B} \mathscr{D}_{t}^{-1}\left(\left(\mathscr{D}_{t} \mathscr{B}^{-1}(M \mathscr{V} \widehat{\varphi}+\bar{M} \overline{\mathscr{V} \widehat{\varphi}})\right)^{3}\right) \\
& =i \xi \widehat{K}(\xi) t^{-1} \mathscr{V}^{*} \bar{M} \mathscr{B}\left(\left(\mathscr{B}^{-1}(M \mathscr{V} \hat{\varphi}+\bar{M} \mathscr{V} \widehat{\varphi})\right)^{3}\right) \\
& =i \xi \widehat{K}(\xi) t^{-1} \mathscr{V}^{*} \frac{1}{\Lambda^{\prime \prime}} \bar{M}(M \mathscr{V} \widehat{\varphi}+\bar{M} \mathscr{V} \widehat{\varphi})^{3} \\
& =i \xi \widehat{K}(\xi) t^{-1} \mathscr{V}^{*} M^{2} \frac{1}{\Lambda^{\prime \prime}}(\mathscr{V} \hat{\varphi})^{3}+3 i \xi \widehat{K}(\xi) t^{-1} \mathscr{V}^{*} \\
& \cdot \frac{1}{\Lambda^{\prime \prime}}(\mathscr{V} \widehat{\varphi})^{2}(\overline{\mathscr{V} \hat{\varphi}})+3 i \xi \widehat{K}(\xi) t^{-1} \mathscr{V}^{*} \bar{M}^{2} \frac{1}{\Lambda^{\prime \prime}}(\mathscr{V} \hat{\varphi}) \\
& \cdot(\overline{\mathscr{V} \widehat{\varphi}})^{2}+i \xi \widehat{K}(\xi) t^{-1} \mathscr{V}^{*} \bar{M}^{4} \frac{1}{\Lambda^{\prime \prime}}(\overline{\mathscr{V} \widehat{\varphi}})^{3} .
\end{aligned}
$$

Note that for $\alpha \neq-1$

$$
\begin{aligned}
\mathscr{V}^{*} & (t) M^{\alpha} \phi=\frac{|t|^{1 / 2}}{\sqrt{2 \pi}} \int_{0}^{\infty} e^{i t S(\eta, \xi)} M^{\alpha} \phi(\eta) \Lambda^{\prime \prime}(\eta) d \eta \\
= & \frac{|t|^{1 / 2}}{\sqrt{2 \pi}} \\
\cdot & e^{i t(\Lambda(\xi)-(1+\alpha) \Lambda(\xi /(1+\alpha)))} \int_{0}^{\infty} e^{i t(1+\alpha) S(\eta, \xi /(1+\alpha))} \phi(\eta) \\
\cdot & \Lambda^{\prime \prime}(\eta) d \eta=e^{i t(\Lambda(\xi)-(1+\alpha) \Lambda(\xi /(1+\alpha)))}|1+\alpha|^{1 / 2} \\
& \cdot \mathscr{D}_{1+\alpha} \mathscr{V}^{*}((1+\alpha) t) \phi .
\end{aligned}
$$

Thus we obtain the following equation for the new dependent variable $\hat{\varphi}(t, \xi)=\mathscr{F} \mathscr{U}(-t) u(t)$ :

$$
\begin{aligned}
\partial_{t} \widehat{\varphi}(t, \xi) & \\
= & \sqrt{3} i \xi \widehat{K}(\xi) t^{-1} e^{i t \Omega(\xi)} \mathscr{D}_{3} \mathscr{V}^{*}(3 t) \frac{1}{\Lambda^{\prime \prime}}(\mathscr{V} \widehat{\varphi})^{3} \\
& +3 i \xi \widehat{K}(\xi) t^{-1} \mathscr{V}^{*}(t) \frac{1}{\Lambda^{\prime \prime}}(\mathscr{V} \widehat{\varphi})^{2}(\overline{\mathscr{V} \widehat{\varphi}}) \\
& +3 i \xi \widehat{K}(\xi) t^{-1} \mathscr{D}_{-1} \mathscr{V}^{*}(-t) \frac{1}{\Lambda^{\prime \prime}}(\mathscr{V} \hat{\varphi})(\overline{\mathscr{V} \widehat{\varphi}})^{2} \\
& +\sqrt{3} i \xi \widehat{K}(\xi) t^{-1} e^{i t \Omega(\xi)} \mathscr{D}_{-3} \mathscr{V}^{*}(-3 t) \frac{1}{\Lambda^{\prime \prime}}(\overline{\mathscr{V} \hat{\varphi}})^{3},
\end{aligned}
$$

where $\Omega(\xi)=\Lambda(\xi)-3 \Lambda(\xi / 3)$.

Now we explain how to use (37) for estimating $|\widehat{\varphi}(t, \xi)|$ uniformly with respect to $\xi$. For the real-valued solution $u$, we have $\overline{\hat{\varphi}(t, \xi)}=\widehat{\varphi}(t,-\xi)$; hence it is sufficient to consider the case $\xi>0$ only. From Lemmas 2 and 3, we find that the last two terms of the right-hand side of (37) are the remainders. We need to consider the first and the second terms of the right-hand side of (37). Due to the oscillating factor $\widehat{K}(\xi) e^{i t \Omega(\xi)}$, integrating by parts with respect to time, we will show that the first term of (37) is also a remainder, since $\widehat{K}(\xi) / \Omega(\xi)$ is bounded in view of the conditions for the symbol $\widehat{K}(\xi)$. 
We organize the rest of our paper as follows. In Section 3, we state main estimates for the decomposition operators $\mathscr{V}(t)$ and $\mathscr{V}^{*}(t)$ related to the evolution group $\mathcal{U}(t)$. We prove a priori estimates of solutions in Section 4. Section 5 is devoted to the proof of Theorem 1 .

\section{Preliminaries}

3.1. Two Kernels. Define the kernel

$$
A_{j}(t, \eta)=\theta(\eta) \sqrt{\frac{t \Lambda^{\prime \prime}(\eta)}{2 \pi}} \int_{0}^{\infty} e^{-i t S(\eta, \xi)} \xi^{j} \chi\left(\xi \eta^{-1}\right) d \xi
$$

where $j=-1,0,1,2$, the phase function $S(\eta, \xi)=\Lambda(\xi)-$ $\Lambda(\eta)-\Lambda^{\prime}(\eta)(\xi-\eta)=(1 / 3) \xi^{-1} \eta^{-2}\left(3 a+2 b \eta^{3} \xi+b \eta^{2} \xi^{2}\right)(\xi-\eta)^{2}$, $\Lambda^{\prime \prime}(\eta)=2 \eta^{-3}\left(a+b \eta^{4}\right)$, the cut-off function $\chi(z) \in \mathbf{C}^{2}(\mathbb{R})$ is such that $\chi(z)=0$ for $z \leq 1 / 3$ or $z \geq 3$ and $\chi(z)=1$ for $2 / 3 \leq z \leq 3 / 2$, and the Heaviside function $\theta(\eta)=1$ for $\eta>0$ and $\theta(\eta)=0$ for $\eta \leq 0$. We change $\xi=\eta y$; then we get

$$
\begin{aligned}
& A_{j}(t, \eta)=\eta^{j} \theta(\eta) \\
& \quad \sqrt{\frac{t \eta^{2} \Lambda^{\prime \prime}(\eta)}{2 \pi}} \int_{0}^{\infty} e^{-i t\left(a y^{-1} \eta^{-1}+(2 / 3) b \eta^{3}+(b / 3) \eta^{3} y\right)(y-1)^{2}} y^{j} \chi(y) d y .
\end{aligned}
$$

To compute the asymptotics of the kernel $A_{j}(t, \eta)$ for large $t$ we apply the stationary phase method (see [21, 22], p. 163):

$$
\begin{aligned}
& \int e^{i t g(y)} f(y) d y \\
& =e^{i t g\left(y_{0}\right)} f\left(y_{0}\right) \sqrt{\frac{2 \pi}{t\left|g^{\prime \prime}\left(y_{0}\right)\right|}} e^{i(\pi / 4) \operatorname{sgn} g^{\prime \prime}\left(y_{0}\right)} \\
& +O\left(t^{-3 / 2}\right)
\end{aligned}
$$

for $t \rightarrow+\infty$, where the stationary point $y_{0}$ is defined by $g^{\prime}\left(y_{0}\right)=0$. By virtue of formula (40) with $g(y)=-\left(a y^{-1} \eta^{-1}+\right.$ $\left.(2 / 3) b \eta^{3}+(b / 3) \eta^{3} y\right)(y-1)^{2}, f(y)=y^{j} \chi(y), y_{0}=1$, we get

$$
A_{j}(t, \eta)=\eta^{j} \theta(\eta)\left(e^{-i(\pi / 4)}+O\left(t^{-1}\right)\right) \text {. }
$$

In particular we have the estimate $\left\|\eta^{-j} A_{j}(t)\right\|_{L^{\infty}} \leq C$. Also we define the kernel

$$
\begin{aligned}
& A^{*}(t, \xi) \\
& \quad=\theta(\xi) \frac{t^{1 / 2}}{\sqrt{2 \pi}} \int_{0}^{\infty} e^{i t S(\eta, \xi)} \chi\left(\eta \xi^{-1}\right) \sqrt{\Lambda^{\prime \prime}(\eta)} d \eta .
\end{aligned}
$$

We change $\eta=\xi y$; then we get

$$
\begin{aligned}
& A^{*}(t, \xi)=\theta(\xi) \frac{t^{1 / 2}}{\sqrt{\pi \xi}} \\
& \quad \cdot \int_{0}^{\infty} e^{i t\left(a \xi^{-1} y^{-2}+(2 b / 3) \xi^{3} y+(b / 3) \xi^{3}\right)(y-1)^{2}} \chi(y) \\
& \cdot \sqrt{y^{-3}\left(a+b \xi^{4} y^{4}\right)} d y .
\end{aligned}
$$

By virtue of formula (40) with $g(y)=\left(a \xi^{-1} y^{-2}+(2 b / 3) \xi^{3} y+\right.$ $\left.(b / 3) \xi^{3}\right)(y-1)^{2}, f(y)=\chi(y) \sqrt{y^{-3}\left(a+b \xi^{4} y^{4}\right)}, y_{0}=1$, we obtain

$$
A^{*}(t, \xi)=\theta(\xi) e^{i(\pi / 4)}+O\left(t^{-1}\right)
$$

In particular we have the estimate $\left\|A^{*}(t)\right\|_{\mathbf{L}^{\infty}} \leq C$.

3.2. Estimates in the Uniform Norm. In the next lemma we estimate the operator $\mathscr{V}$ in the uniform norm. Denote $\mu_{-1}=$ $5 / 4, \mu_{0}=1 / 4, \mu_{1}=0, \nu_{-1}=-5 / 4, v_{0}=1$, and $\nu_{1}=1 / 4$.

Lemma 2. Let $j=-1,0,1$. Then the estimates

$$
\begin{gathered}
\left\||\eta|^{\mu_{j}}\langle\eta\rangle^{v_{j}}\left(\mathscr{V} \xi^{j} \phi-A_{j}(t) \phi\right)\right\|_{\mathbf{L}^{\infty}(0, \infty)} \\
\leq C t^{-1 / 2}\left\||\xi|^{1 / 2} \phi\right\|_{\mathbf{L}^{\infty}}+C t^{-1 / 4}\left\|\xi \phi_{\xi}\right\|_{\mathbf{L}^{2}} \\
\left\||\eta|^{v_{j}}\langle\eta\rangle^{-2 v_{j}+1} \mathscr{V} \xi^{j} \phi\right\|_{\mathbf{L}^{\infty}(-\infty, 0)} \\
\leq C t^{-1 / 2}\left(\left\||\xi|^{1 / 2} \phi\right\|_{\mathbf{L}^{\infty}}+\left\|\xi \phi_{\xi}\right\|_{\mathbf{L}^{2}}\right)
\end{gathered}
$$

are valid for all $t \geq 1$.

Proof. We write

$$
\begin{aligned}
& \mathscr{V} \xi^{j} \phi-A_{j}(t, \eta) \phi(\eta) \\
& =\sqrt{\frac{t \Lambda^{\prime \prime}(\eta)}{2 \pi}} \int_{0}^{\infty} e^{-i t S(\eta, \xi)}(\phi(\xi)-\phi(\eta)) \\
& \quad \cdot \xi^{j} \chi\left(\xi \eta^{-1}\right) d \xi+\sqrt{\frac{t \Lambda^{\prime \prime}(\eta)}{2 \pi}} \int_{0}^{\infty} e^{-i t S(\eta, \xi)} \phi(\xi) \\
& \quad \cdot\left(1-\chi\left(\xi \eta^{-1}\right)\right) \xi^{j} d \xi=I_{1}+I_{2}
\end{aligned}
$$

for $\eta>0$. For the first summand $I_{1}$ we integrate by parts via the identity

$$
e^{-i t S(\eta, \xi)}=H_{1} \partial_{\xi}\left((\xi-\eta) e^{-i t S(\eta, \xi)}\right)
$$

with $H_{1}=\left(1-i t(\xi-\eta) \partial_{\xi} S(\eta, \xi)\right)^{-1}, \partial_{\xi} S(\eta, \xi)=\xi^{-2} \eta^{-2}(a+$ $\left.b \eta^{2} \xi^{2}\right)\left(\xi^{2}-\eta^{2}\right)$, to get

$$
\begin{aligned}
I_{1} & =C \sqrt{t \Lambda^{\prime \prime}(\eta)} \int_{0}^{\infty} e^{-i t S(\eta, \xi)}(\phi(\xi)-\phi(\eta))(\xi-\eta) \\
& \cdot \partial_{\xi}\left(\xi^{j} H_{1} \chi\left(\xi \eta^{-1}\right)\right)+e^{-i t S(\eta, \xi)}(\xi-\eta) \\
& \cdot \xi^{j} H_{1} \chi\left(\xi \eta^{-1}\right) \phi_{\xi}(\xi) d \xi .
\end{aligned}
$$


Using the estimates

$$
\begin{aligned}
& |\phi(\xi)-\phi(\eta)| \leq C \eta^{-1} \int_{\xi}^{\eta} \xi \phi_{\xi}(\xi) d \xi \\
& \leq C \eta^{-1}|\xi-\eta|^{1 / 2}\left\|\xi \phi_{\xi}\right\|_{L^{2}}, \\
& \left|(\xi-\eta) \xi^{j} H_{1} \chi\left(\xi \eta^{-1}\right)\right| \leq \frac{C|\xi-\eta| \eta^{j}}{1+t \eta^{-3}\langle\eta\rangle^{4}(\xi-\eta)^{2}} \\
& \left|(\xi-\eta) \partial_{\xi}\left(\xi^{j} H_{1} \chi\left(\xi \eta^{-1}\right)\right)\right| \leq \frac{C \eta^{j}}{1+t \eta^{-3}\langle\eta\rangle^{4}(\xi-\eta)^{2}}
\end{aligned}
$$

in the domain $0<(1 / 3) \eta \leq \xi \leq 3 \eta$, we find

$$
\begin{aligned}
& \left|I_{1}\right| \leq C t^{1 / 2} \eta^{j-5 / 2}\langle\eta\rangle^{2}\left\|\xi \phi_{\xi}\right\|_{L^{2}} \\
& \quad \cdot \int_{(1 / 3) \eta}^{3 \eta} \frac{|\xi-\eta|^{1 / 2} d \xi}{1+t \eta^{-3}\langle\eta\rangle^{4}(\xi-\eta)^{2}}+C t^{1 / 2} \eta^{j-5 / 2}\langle\eta\rangle^{2} \\
& \cdot \int_{(1 / 3) \eta}^{3 \eta} \frac{|\xi-\eta|\left|\xi \phi_{\xi}(\xi)\right| d \xi}{1+t \eta^{-3}\langle\eta\rangle^{4}(\xi-\eta)^{2}} \leq C t^{1 / 2} \eta^{j-5 / 2}\langle\eta\rangle^{2} \\
& \cdot\left\|\xi \phi_{\xi}\right\|_{L^{2}}\left(\int_{(1 / 3) \eta}^{3 \eta} \frac{|\xi-\eta|^{1 / 2} d \xi}{1+t \eta^{-3}\langle\eta\rangle^{4}(\xi-\eta)^{2}}\right. \\
& \left.+\left(\int_{(1 / 3) \eta}^{3 \eta} \frac{(\xi-\eta)^{2} d \xi}{\left(1+t \eta^{-3}\langle\eta\rangle^{4}(\xi-\eta)^{2}\right)^{2}}\right)^{1 / 2}\right) .
\end{aligned}
$$

Changing $\xi=\eta y$ we have

$$
\begin{gathered}
\int_{(1 / 3) \eta}^{3 \eta} \frac{|\xi-\eta|^{1 / 2} d \xi}{1+t \eta^{-3}\langle\eta\rangle^{4}(\xi-\eta)^{2}} \\
\leq C \eta^{3 / 2} \int_{1 / 3}^{3} \frac{|y-1|^{1 / 2} d y}{1+t \eta^{-1}\langle\eta\rangle^{4}(y-1)^{2}} \\
\leq C \eta^{3 / 2}\left\langle t \eta^{-1}\langle\eta\rangle^{4}\right\rangle^{-3 / 4} \leq C t^{-3 / 4} \eta^{9 / 4}\langle\eta\rangle^{-3} \\
\int_{(1 / 3) \eta}^{3 \eta} \frac{(\xi-\eta)^{2} d \xi}{\left(1+t \eta^{-3}\langle\eta\rangle^{4}(\xi-\eta)^{2}\right)^{2}} \\
\leq C \eta^{3} \int_{1 / 3}^{3} \frac{(y-1)^{2} d y}{\left(1+t \eta^{-1}\langle\eta\rangle^{4}(y-1)^{2}\right)^{2}} \\
\leq C \eta^{3}\left\langle t \eta^{-1}\langle\eta\rangle^{4}\right\rangle^{-3 / 2} \leq C t^{-3 / 2} \eta^{9 / 2}\langle\eta\rangle^{-6} .
\end{gathered}
$$

Thus we obtain

$$
\left|I_{1}\right| \leq C t^{-1 / 4} \eta^{j-1 / 4}\langle\eta\rangle^{-1}\left\|\xi \phi_{\xi}\right\|_{\mathbf{L}^{2}}
$$

for all $t \geq 1, \eta>0$, and $j=-1,0,1$.
To estimate the second integral $I_{2}$ we integrate by parts via the identity

$$
e^{-i t S(x, \xi)}=H_{2} \partial_{\xi}\left(\xi e^{-i t S(x, \xi)}\right)
$$

with $H_{2}=\left(1-i t \xi \partial_{\xi} S(\eta, \xi)\right)^{-1}, \partial_{\xi} S(\eta, \xi)=\xi^{-2} \eta^{-2}(a+$ $\left.b \eta^{2} \xi^{2}\right)\left(\xi^{2}-\eta^{2}\right)$, to get

$$
\begin{aligned}
I_{2} & =C \sqrt{t \Lambda^{\prime \prime}(\eta)} \int_{0}^{\infty} e^{-i t S(\eta, \xi)} \phi(\xi) \\
& \cdot \xi \partial_{\xi}\left(\left(1-\chi\left(\xi \eta^{-1}\right)\right) H_{2} \xi^{j}\right) \\
& +e^{-i t S(x, \xi)} H_{2}\left(1-\chi\left(\xi \eta^{-1}\right)\right) \xi^{j+1} \phi_{\xi}(\xi) d \xi .
\end{aligned}
$$

Using the estimate

$$
\begin{aligned}
& \left|H_{2}\left(1-\chi\left(\xi \eta^{-1}\right)\right) \xi^{j}\right|+\left|\xi \partial_{\xi}\left(\left(1-\chi\left(\xi \eta^{-1}\right)\right) H_{2} \xi^{j}\right)\right| \\
& \quad \leq \frac{C \xi^{j}}{1+t \xi^{-1} \eta^{-2}\left(1+\xi^{2} \eta^{2}\right)\left(\xi^{2}+\eta^{2}\right)}
\end{aligned}
$$

in the domain $0<\xi \leq(2 / 3) \eta$, or $\xi \geq(3 / 2) \eta>0$, we obtain

$$
\begin{aligned}
& \left|I_{2}\right| \leq C t^{1 / 2} \eta^{-3 / 2}\langle\eta\rangle^{2} \\
& \quad \cdot \int_{0}^{\infty} \frac{\xi^{j}\left(|\phi(\xi)|+\left|\xi \phi_{\xi}(\xi)\right|\right) d \xi}{1+t \xi^{-1} \eta^{-2}\left(1+\xi^{2} \eta^{2}\right)\left(\xi^{2}+\eta^{2}\right)} \\
& \quad \leq C t^{1 / 2} \eta^{-3 / 2}\langle\eta\rangle^{2}\left\|\xi^{1 / 2} \phi\right\|_{L^{\infty}} \\
& \quad \cdot \int_{0}^{\infty} \frac{\xi^{j-1 / 2} d \xi}{1+t \xi^{-1} \eta^{-2}\left(1+\xi^{2} \eta^{2}\right)\left(\xi^{2}+\eta^{2}\right)} \\
& +C t^{1 / 2} \eta^{-3 / 2}\langle\eta\rangle^{2}\left\|\xi \phi_{\xi}\right\|_{L^{2}} \\
& \quad \cdot\left(\int_{0}^{\infty} \frac{\xi^{2 j} d \xi}{\left(1+t \xi^{-1} \eta^{-2}\left(1+\xi^{2} \eta^{2}\right)\left(\xi^{2}+\eta^{2}\right)\right)^{2}}\right)^{1 / 2} .
\end{aligned}
$$

Changing $\xi=\eta y$ we obtain

$$
\begin{aligned}
& \int_{0}^{\infty} \frac{\xi^{j-1 / 2} d \xi}{1+t \xi^{-1} \eta^{-2}\left(1+\xi^{2} \eta^{2}\right)\left(\xi^{2}+\eta^{2}\right)} \\
& \quad \leq \int_{0}^{\infty} \frac{\eta^{j+1 / 2} y^{j-1 / 2} d y}{1+t \eta^{-1} y^{-1}\left(1+\eta^{4} y^{2}\right)\langle y\rangle^{2}} .
\end{aligned}
$$

For $\eta \leq 1$

$$
\begin{aligned}
& \int_{0}^{1} \frac{\eta^{j+1 / 2} y^{j-1 / 2} d y}{1+t \eta^{-1} y^{-1}\left(1+\eta^{4} y^{2}\right)} \leq C t^{-1} \eta^{j+3 / 2} \int_{0}^{1} y^{j+1 / 2} d y \\
& \quad \leq C t^{-1} \eta^{j+3 / 2}, \\
& \int_{1}^{\infty} \frac{\eta^{j+1 / 2} y^{j-1 / 2} d y}{1+t \eta^{-1} y\left(1+\eta^{4} y^{2}\right)} \leq C t^{-1} \eta^{j+3 / 2} \int_{1}^{\infty} \frac{y^{j-3 / 2} d y}{1+\eta^{4} y^{2}} \\
& \quad \leq C t^{-1} \eta^{3 / 2-v_{j}}
\end{aligned}
$$


International Journal of Differential Equations

7

$\nu_{-1}=1$, and $v_{0}=v_{1}=0$. For $\eta>1$

$$
\begin{aligned}
& \int_{0}^{1} \frac{\eta^{j+1 / 2} y^{j-1 / 2} d y}{1+t \eta^{-1} y^{-1}\left(1+\eta^{4} y^{2}\right)} \\
& \leq C t^{-1} \eta^{j+3 / 2} \int_{0}^{1 / \eta^{2}} y^{j+1 / 2} d y \\
& \quad+C t^{-1} \eta^{j-5 / 2} \int_{1 / \eta^{2}}^{1} y^{j-3 / 2} d y \\
& \leq C t^{-1}\langle\eta\rangle^{-j-3 / 2}+C t^{-1}\langle\eta\rangle^{j-5 / 2} \\
& \int_{1}^{\infty} \frac{\eta^{j+1 / 2} y^{j-1 / 2} d y}{1+t \eta^{-1} y\left(1+\eta^{4} y^{2}\right)} \leq C t^{-1} \eta^{j-5 / 2} \int_{1}^{\infty} y^{j-7 / 2} d y \\
& \leq C t^{-1}\langle\eta\rangle^{j-5 / 2} .
\end{aligned}
$$

Hence

$$
\begin{gathered}
\eta^{-3 / 2}\langle\eta\rangle^{2} \int_{0}^{\infty} \frac{\xi^{j-1 / 2} d \xi}{1+t \xi^{-1} \eta^{-2}\left(1+\xi^{2} \eta^{2}\right)\left(\xi^{2}+\eta^{2}\right)} \\
\leq C t^{-1}\left(\{\eta\}^{j}+\{\eta\}^{-v_{j}}\right)\left(\langle\eta\rangle^{-j-1}+\langle\eta\rangle^{j-2}\right) \\
\leq C t^{-1}\{\eta\}^{-v_{j}}\langle\eta\rangle^{v_{j}-1} \leq C t^{-1} \eta^{-v_{j}}\langle\eta\rangle^{2 v_{j}-1} .
\end{gathered}
$$

In the same manner changing $\xi=\eta y$ we get

$$
\begin{aligned}
& \int_{0}^{\infty} \frac{\xi^{2 j} d \xi}{\left(1+t \xi^{-1} \eta^{-2}\left(1+\xi^{2} \eta^{2}\right)\left(\xi^{2}+\eta^{2}\right)\right)^{2}} \\
& \quad \leq C \int_{0}^{\infty} \frac{\eta^{2 j+1} y^{2 j} d y}{\left(1+t \eta^{-1} y^{-1}\left(1+\eta^{4} y^{2}\right)\langle y\rangle^{2}\right)^{2}} .
\end{aligned}
$$

For $\eta \leq 1$

$$
\begin{aligned}
& \int_{0}^{1} \frac{\eta^{2 j+1} y^{2 j} d y}{\left(1+t \eta^{-1} y^{-1}\left(1+\eta^{4} y^{2}\right)\right)^{2}} \\
& \quad \leq C t^{-2} \eta^{2 j+3} \int_{0}^{1} y^{2 j+2} d y \leq C t^{-2} \eta^{2 j+3} \leq C t^{-2} \eta^{3-2 v_{j}} \\
& \int_{1}^{\infty} \frac{\eta^{2 j+1} y^{2 j} d y}{\left(1+t \eta^{-1} y\left(1+\eta^{4} y^{2}\right)\right)^{2}} \\
& \quad \leq C t^{-2} \eta^{2 j+3} \int_{1}^{\infty} \frac{y^{2 j-2} d y}{\left(1+\eta^{4} y^{2}\right)^{2}} \leq C t^{-2} \eta^{2 j+3-2(2 j-1)} \\
& \quad \leq C t^{-2} \eta^{3-2 v_{j}}
\end{aligned}
$$

$v_{-1}=1$, and $v_{0}=v_{1}=0$. For $\eta>1$

$$
\begin{aligned}
& \int_{0}^{1} \frac{\eta^{2 j+1} y^{2 j} d y}{\left(1+t \eta^{-1} y^{-1}\left(1+\eta^{4} y^{2}\right)\right)^{2}} \\
& \leq C t^{-2} \eta^{2 j+3} \int_{0}^{1 / \eta^{2}} y^{2 j+2} d y \\
&+C t^{-2} \eta^{2 j-5} \int_{1 / \eta^{2}}^{1} y^{2 j-2} d y \\
& \leq C t^{-2}\langle\eta\rangle^{-2 j-3}+C t^{-2}\langle\eta\rangle^{2 j-5} \\
& \int_{1}^{\infty} \quad \frac{\eta^{2 j+1} y^{2 j} d y}{\left(1+t \eta^{-1} y\left(1+\eta^{4} y^{2}\right)\right)^{2}} \leq C t^{-2} \eta^{2 j-5} \int_{1}^{\infty} y^{2 j-6} d y \\
& \leq C t^{-2}\langle\eta\rangle^{2 j-5} .
\end{aligned}
$$

Hence

$$
\begin{aligned}
\eta^{-3} & \langle\eta\rangle^{4} \int_{0}^{\infty} \frac{\xi^{2 j} d \xi}{\left(1+t \xi^{-1} \eta^{-2}\left(1+\xi^{2} \eta^{2}\right)\left(\xi^{2}+\eta^{2}\right)\right)^{2}} \\
& \leq C t^{-2}\{\eta\}^{-2 v_{j}}\left(\langle\eta\rangle^{-2 j-2}+\langle\eta\rangle^{2 j-4}\right) \\
& \leq C t^{-2}\{\eta\}^{-2 v_{j}}\langle\eta\rangle^{2 v_{j}-2} \leq C t^{-2} \eta^{-2 v_{j}}\langle\eta\rangle^{4 v_{j}-2}
\end{aligned}
$$

for $j=-1,0,1$. Thus we have

$$
\left|I_{2}\right| \leq C t^{-1 / 2} \eta^{-v_{j}}\langle\eta\rangle^{2 v_{j}-1}\left(\left\|\xi \phi_{\xi}\right\|_{\mathbf{L}^{2}}+\left\|\xi^{1 / 2} \phi\right\|_{\mathbf{L}^{\infty}}\right)
$$

for all $t \geq 1, \eta>0$, and $j=-1,0,1$.

For the case of $\eta<0$ we integrate by parts using identity (53):

$$
\begin{aligned}
& \mathscr{V} \xi^{j} \phi=C \sqrt{t \Lambda^{\prime \prime}(\eta)} \int_{0}^{\infty} e^{-i t S(\eta, \xi)} \phi(\xi) \xi \partial_{\xi}\left(H_{2} \xi^{j}\right) \\
& +e^{-i t S(x, \xi)} H_{2} \xi^{j} \xi \phi_{\xi}(\xi) d \xi
\end{aligned}
$$

Using the estimate

$$
\begin{aligned}
& \left|H_{2} \xi^{j}\right|+\left|\xi \partial_{\xi}\left(H_{2} \xi^{j}\right)\right| \\
& \quad \leq \frac{C \xi^{j}}{1+t \xi^{-1}|\eta|^{-2}\left(1+\xi^{2} \eta^{2}\right)\left(\xi^{2}+\eta^{2}\right)}
\end{aligned}
$$

in the domain $\xi>0, \eta<0$, we obtain

$$
\begin{aligned}
& \left|\mathscr{V} \xi^{j} \phi\right| \leq C t^{1 / 2}|\eta|^{-3 / 2}\langle\eta\rangle^{2} \\
& \cdot \int_{0}^{\infty} \frac{\xi^{j}\left(|\phi(\xi)|+\left|\xi \phi_{\xi}(\xi)\right|\right) d \xi}{1+t \xi^{-1}|\eta|^{-2}\left(1+\xi^{2} \eta^{2}\right)\left(\xi^{2}+\eta^{2}\right)} \\
& \quad \leq C t^{1 / 2}|\eta|^{-3 / 2}\langle\eta\rangle^{2}\left\|\xi^{1 / 2} \phi\right\|_{L^{\infty}}
\end{aligned}
$$




$$
\begin{aligned}
& \int_{0}^{\infty} \frac{\xi^{j-1 / 2} d \xi}{1+t \xi^{-1}|\eta|^{-2}\left(1+\xi^{2} \eta^{2}\right)\left(\xi^{2}+\eta^{2}\right)} \\
& +C t^{1 / 2}|\eta|^{-3 / 2}\langle\eta\rangle^{2}\left\|\xi \phi_{\xi}\right\|_{L^{2}} \\
& \cdot\left(\int_{0}^{\infty} \frac{\xi^{2 j} d \xi}{\left(1+t \xi^{-1}|\eta|^{-2}\left(1+\xi^{2} \eta^{2}\right)\left(\xi^{2}+\eta^{2}\right)\right)^{2}}\right)^{1 / 2} .
\end{aligned}
$$

Then as above we get

$$
\begin{aligned}
& \left|\mathscr{V} \xi^{j} \phi\right| \\
& \quad \leq C t^{-1 / 2}|\eta|^{-v_{j}}\langle\eta\rangle^{2 v_{j}-1}\left(\left\|\xi \phi_{\xi}\right\|_{\mathbf{L}^{2}}+\left\||\xi|^{1 / 2} \phi\right\|_{\mathbf{L}^{\infty}}\right)
\end{aligned}
$$

for all $t \geq 1, \eta<0$, and $j=-1,0,1$. Lemma 2 is proved.

By Lemma 2, we have the estimate

$$
\begin{aligned}
\left|\mathscr{V} \xi^{j} \phi\right| & \leq C|\eta|^{j}|\phi(\eta)| \\
& +C t^{-1 / 4}|\eta|^{-\mu_{j}}\langle\eta\rangle^{-v_{j}}\left(\left\||\xi|^{1 / 2} \phi\right\|_{\mathbf{L}^{\infty}}+\left\|\xi \phi_{\xi}\right\|_{\mathbf{L}^{2}}\right) \\
\leq & C|\eta|^{j-1 / 2}\left\||\xi|^{1 / 2} \phi\right\|_{\mathbf{L}^{\infty}} \\
& +C t^{-1 / 4}|\eta|^{-\mu_{j}}\langle\eta\rangle^{-v_{j}}\left(\left\||\xi|^{1 / 2} \phi\right\|_{\mathbf{L}^{\infty}}+\left\|\xi \phi_{\xi}\right\|_{\mathbf{L}^{2}}\right) .
\end{aligned}
$$

We next consider the operator $\mathscr{V}^{*}$.

Lemma 3. The estimates

$$
\begin{aligned}
& \left\|\langle\xi\rangle\left(\mathscr{V}^{*} \phi-A^{*}(t, \xi) \phi(\xi)\right)\right\|_{\mathbf{L}^{\infty}(0, \infty)} \\
& \quad \leq C t^{-1 / 4}\left(\|\phi\|_{\mathbf{L}^{\infty}}+\left\||\eta|^{-9 / 4}\langle\eta\rangle^{4} t \mathscr{A}_{0} \phi\right\|_{\mathbf{L}^{2}}\right), \\
& \left\|\langle\xi\rangle \mathscr{V}^{*} \phi\right\|_{\mathbf{L}^{\infty}(-\infty, 0)} \\
& \quad \leq C t^{-1 / 2}\left(\|\phi\|_{\mathbf{L}^{\infty}}+\left\||\eta|^{-9 / 4}\langle\eta\rangle^{4} t \mathscr{A}_{0} \phi\right\|_{\mathbf{L}^{2}}\right)
\end{aligned}
$$

are valid for all $t \geq 1$, where $\mathscr{A}_{0}=\left(1 / t \sqrt{\Lambda^{\prime \prime}}\right) \partial_{\eta}\left(1 / \sqrt{\Lambda^{\prime \prime}}\right)$.

Proof. We find

$$
\begin{aligned}
& \mathscr{V}^{*} \phi-A^{*}(t, \xi) \phi(\xi)=\frac{t^{1 / 2}}{\sqrt{2 \pi}} \\
& \quad \int_{0}^{\infty} e^{i t S(\eta, \xi)}(\phi(\eta)-\phi(\xi)) \chi\left(\eta \xi^{-1}\right) \sqrt{\Lambda^{\prime \prime}(\eta)} d \eta \\
& \quad+\frac{t^{1 / 2}}{\sqrt{2 \pi}} \int_{0}^{\infty} e^{i t S(\eta, \xi)} \phi(\eta)\left(1-\chi\left(\eta \xi^{-1}\right)\right) \sqrt{\Lambda^{\prime \prime}(\eta)} d \eta \\
& \quad=I_{3}+I_{4}
\end{aligned}
$$

for $\xi>0$. In the first integral $I_{3}$ using the identity

$$
e^{i t S(x, \xi)}=H_{3} \partial_{\eta}\left((\eta-\xi) e^{i t S(\eta, \xi)}\right)
$$

with $H_{3}=\left(1+i t(\eta-\xi) S_{\eta}(\eta, \xi)\right)^{-1}, \partial_{\eta} S(\eta, \xi)=2 \eta^{-3}\left(a+b \eta^{4}\right)(\eta-$ $\xi)$, we integrate by parts

$$
\begin{aligned}
I_{3} & =C t^{1 / 2} \int_{0}^{\infty} e^{i t S(\eta, \xi)}(\phi(\eta)-\phi(\xi))(\eta-\xi) \\
& \cdot \partial_{\eta}\left(H_{3} \chi\left(\eta \xi^{-1}\right) \sqrt{\Lambda^{\prime \prime}(\eta)}\right)+e^{i t S(\eta, \xi)} \phi_{\eta}(\eta)(\eta-\xi) \\
& \cdot H_{3} \chi\left(\eta \xi^{-1}\right) \sqrt{\Lambda^{\prime \prime}(\eta)} d \eta .
\end{aligned}
$$

Then using the identity

$$
\phi_{\eta}(\eta)=\Lambda^{\prime \prime}(\eta) t \mathscr{A}_{0} \phi(\eta)+\frac{\Lambda^{\prime \prime \prime}(\eta)}{2 \Lambda^{\prime \prime}(\eta)} \phi(\eta)
$$

we get

$$
\begin{aligned}
I_{3}= & C t^{1 / 2} \int_{0}^{\infty} e^{i t S(\eta, \xi)}(\phi(\eta)-\phi(\xi))(\eta-\xi) \\
& \cdot \partial_{\eta}\left(H_{3} \chi\left(\eta \xi^{-1}\right) \sqrt{\Lambda^{\prime \prime}(\eta)}\right)+e^{i t S(\eta, \xi)}(\eta-\xi) \\
& \cdot H_{3} \chi\left(\eta \xi^{-1}\right)\left(\Lambda^{\prime \prime}(\eta)\right)^{3 / 2} t \mathscr{A}_{0} \phi(\eta)+e^{i t S(\eta, \xi)} \\
& \cdot \frac{\Lambda^{\prime \prime \prime}(\eta)}{\sqrt{\Lambda^{\prime \prime}(\eta)}} \phi(\eta)(\eta-\xi) H_{3} \chi\left(\eta \xi^{-1}\right) d \eta .
\end{aligned}
$$

Applying the estimates

$$
\begin{aligned}
& |\phi(\eta)-\phi(\xi)|=\left|\int_{\eta}^{\xi} \partial_{\eta} \phi d \eta\right| \\
& =\left|\int_{\eta}^{\xi} \Lambda^{\prime \prime}(\eta) t \mathscr{A}_{0} \phi(\eta) d \eta+\int_{\eta}^{\xi} \frac{\Lambda^{\prime \prime \prime}(\eta)}{2 \Lambda^{\prime \prime}(\eta)} \phi(\eta) d \eta\right| \\
& \leq C|\eta-\xi|^{1 / 2}|\xi|^{9 / 4}\langle\xi\rangle^{-3} \Lambda^{\prime \prime}(\xi) \\
& \cdot\left\||\eta|^{-9 / 4}\langle\eta\rangle^{3} t \mathscr{A}_{0} \phi\right\|_{L^{2}}+C|\xi|^{-3 / 2}\langle\xi\rangle^{2}|\eta-\xi| \\
& \cdot\left\||\eta|^{1 / 2}\langle\eta\rangle^{-2} \phi\right\|_{L^{\infty}}, \\
& \left|H_{3}\right| \leq C\left(1+t \xi^{-3}\langle\xi\rangle^{4}(\xi-\eta)^{2}\right)^{-1} \\
& \left|(\eta-\xi) H_{3} \chi\left(\eta \xi^{-1}\right)\left(\Lambda^{\prime \prime}(\eta)\right)^{3 / 2}\right| \\
& \leq \frac{C \xi^{-9 / 2}\langle\xi\rangle^{6}|\eta-\xi|}{1+t \xi^{-3}\langle\xi\rangle^{4}(\xi-\eta)^{2}} \\
& \text { and } \Lambda^{\prime \prime}(\xi)=O\left(\xi^{-3}\langle\xi\rangle^{4}\right), \Lambda^{\prime \prime \prime}(\xi)=O\left(\xi^{-4}\langle\xi\rangle^{4}\right), \text { and } \\
& \left|(\eta-\xi) \partial_{\eta}\left(H_{3} \chi\left(\eta \xi^{-1}\right) \sqrt{\Lambda^{\prime \prime}(\eta)}\right)\right| \\
& \mid \xi^{-3}\langle\xi\rangle^{4}(\xi-\eta)^{2}
\end{aligned}
$$


for $(1 / 3) \xi \leq \eta \leq 3 \xi$, we find

$$
\begin{aligned}
\left|I_{3}\right| \leq C & t^{1 / 2}|\xi|^{-9 / 4}\langle\xi\rangle^{3}\left\||\eta|^{-9 / 4}\langle\eta\rangle^{3} t \mathscr{A}_{0} \phi\right\|_{L^{2}} \\
& \cdot \int_{(1 / 3) \xi}^{3 \xi} \frac{|\eta-\xi|^{1 / 2} d \eta}{1+t \xi^{-3}\langle\xi\rangle^{4}(\xi-\eta)^{2}}+C t^{1 / 2} \xi^{-3}\langle\xi\rangle^{4} \\
& \cdot\left\||\eta|^{1 / 2}\langle\eta\rangle^{-2} \phi\right\|_{L^{\infty}} \\
& \cdot \int_{(1 / 3) \xi}^{3 \xi} \frac{|\eta-\xi| d \eta}{1+t \xi^{-3}\langle\xi\rangle^{4}(\xi-\eta)^{2}}+C t^{1 / 2}|\xi|^{-9 / 4} \\
& \cdot\langle\xi\rangle^{3}\left\||\eta|^{-9 / 4}\langle\eta\rangle^{3} t \mathscr{A}_{0} \phi\right\|_{L^{2}} \\
& \cdot\left(\int_{(1 / 3) \xi}^{3 \xi} \frac{(\eta-\xi)^{2} d \eta}{\left(1+t \xi^{-3}\langle\xi\rangle^{4}(\xi-\eta)^{2}\right)^{2}}\right)^{1 / 2} .
\end{aligned}
$$

Hence

$$
\begin{aligned}
\left|I_{3}\right| & \leq C t^{1 / 2}|\xi|^{-9 / 4}\langle\xi\rangle^{2}\left\langle t \xi^{-3}\langle\xi\rangle^{4}\right\rangle^{-3 / 4} \\
\cdot & \left\||\eta|^{-9 / 4}\langle\eta\rangle^{4} t \mathscr{A}_{0} \phi\right\|_{L^{2}}+C t^{1 / 2} \xi^{-5 / 2}\langle\xi\rangle^{3} \\
\cdot & \left\langle t \xi^{-3}\langle\xi\rangle^{4}\right\rangle^{\gamma-1}\|\phi\|_{L^{\infty}} \leq C t^{-1 / 4}\langle\xi\rangle^{-1} \\
\cdot & \left\||\eta|^{-9 / 4}\langle\eta\rangle^{3} t \mathscr{A}_{0} \phi\right\|_{L^{2}}+C t^{\gamma-1 / 2}\langle\xi\rangle^{-1}\|\phi\|_{L^{\infty}} .
\end{aligned}
$$

In the second integral $I_{4}$, using the identity $e^{i t S(\eta, \xi)}=$ $H_{4} \partial_{\eta}\left(\eta e^{i t S(\eta, \xi)}\right)$ with $H_{4}=\left(1+i t \eta S_{\eta}(\eta, \xi)\right)^{-1}$ we integrate by parts

$$
\begin{aligned}
I_{4} & =C t^{1 / 2} \int_{0}^{\infty} e^{i t S(\eta, \xi)} \phi(\eta) \\
& \cdot \eta \partial_{\eta}\left(H_{4}\left(1-\chi\left(\eta \xi^{-1}\right)\right) \sqrt{\Lambda^{\prime \prime}(\eta)}\right) \\
& +e^{i t S(\eta, \xi)} \phi_{\eta}(\eta) \eta H_{4}\left(1-\chi\left(\eta \xi^{-1}\right)\right) \sqrt{\Lambda^{\prime \prime}(\eta)} d \eta .
\end{aligned}
$$

Then using

$$
\phi_{\eta}(\eta)=\Lambda^{\prime \prime}(\eta) t \mathscr{A}_{0} \phi(\eta)+\frac{\Lambda^{\prime \prime \prime}(\eta)}{2 \Lambda^{\prime \prime}(\eta)} \phi(\eta)
$$

we get

$$
\begin{aligned}
\left|I_{4}\right| & \leq C t^{1 / 2} \int_{0}^{\infty}|\phi(\eta)| \\
& \cdot\left|\eta \partial_{\eta}\left(H_{4}\left(1-\chi\left(\eta \xi^{-1}\right)\right) \sqrt{\Lambda^{\prime \prime}(\eta)}\right)\right| \\
& +\left|\frac{\Lambda^{\prime \prime \prime}(\eta)}{\sqrt{\Lambda^{\prime \prime}(\eta)}} \eta H_{4}\left(1-\chi\left(\eta \xi^{-1}\right)\right)\right||\phi(\eta)| \\
& +\left|\left(\Lambda^{\prime \prime}(\eta)\right)^{3 / 2} \eta H_{4}\left(1-\chi\left(\eta \xi^{-1}\right)\right)\right| \\
& \cdot\left|t \mathscr{A}_{0} \phi(\eta)\right| d \eta .
\end{aligned}
$$

Then using the estimates

$$
\begin{aligned}
& \left|\eta \partial_{\eta}\left(H_{4}\left(1-\chi\left(\eta \xi^{-1}\right)\right) \sqrt{\Lambda^{\prime \prime}(\eta)}\right)\right| \\
& +\left|\frac{\Lambda^{\prime \prime \prime}(\eta)}{\sqrt{\Lambda^{\prime \prime}(\eta)}} \eta H_{4}\left(1-\chi\left(\eta \xi^{-1}\right)\right)\right| \\
& \leq \frac{C \eta^{-3 / 2}\langle\eta\rangle^{2}}{1+t \eta^{-2}\langle\eta\rangle^{4}(\xi+\eta)}, \\
& \left|\left(\Lambda^{\prime \prime}(\eta)\right)^{3 / 2} \eta H_{4}\left(1-\chi\left(\eta \xi^{-1}\right)\right)\right| \\
& \leq \frac{C \eta^{-7 / 2}\langle\eta\rangle^{6}}{1+t \eta^{-2}\langle\eta\rangle^{4}(\xi+\eta)}
\end{aligned}
$$

in the domain $\eta \geq 3 \xi>0$ or $0<\eta<(1 / 3) \xi$, we get

$$
\begin{aligned}
\left|I_{4}\right| \leq C t^{1 / 2}\|\phi\|_{L^{\infty}} \int_{0}^{\infty} \frac{\eta^{-3 / 2}\langle\eta\rangle^{2} d \eta}{1+t \eta^{-2}\langle\eta\rangle^{4}(\xi+\eta)} \\
+C t^{1 / 2}\left\||\eta|^{-9 / 4}\langle\eta\rangle^{4} t \mathscr{A}_{0} \phi\right\|_{\mathbf{L}^{2}} \\
+\left(\int_{0}^{\infty} \frac{\eta^{-5 / 2}\langle\eta\rangle^{5} d \eta}{\left(1+t \eta^{-2}\langle\eta\rangle^{4}(\xi+\eta)\right)^{2}}\right)^{1 / 2} .
\end{aligned}
$$

Therefore

$$
\begin{aligned}
\left|I_{4}\right| & \leq C t^{-1 / 2}\|\phi\|_{L^{\infty}} \int_{0}^{\infty} \frac{\eta^{1 / 2}\langle\eta\rangle^{-2} d \eta}{\xi+\eta} \\
& +C t^{-1 / 2}\left\||\eta|^{-9 / 4}\langle\eta\rangle^{4} t \mathscr{A}_{0} \phi\right\|_{L^{2}} \\
& \cdot\left(\int_{0}^{\infty} \frac{\eta^{3 / 2}\langle\eta\rangle^{-3} d \eta}{(\xi+\eta)^{2}}\right)^{1 / 2} \leq C t^{-1 / 2}\langle\xi\rangle^{-1}\|\phi\|_{L^{\infty}} \\
& +C t^{-1 / 2}\langle\xi\rangle^{-1}\left\||\eta|^{-9 / 4}\langle\eta\rangle^{4} t \mathscr{A}_{0} \phi\right\|_{L^{2}} .
\end{aligned}
$$

Next we consider $\xi<0$. Using the identity $e^{i t S(\eta, \xi)}=$ $H_{4} \partial_{\eta}\left(\eta e^{i t S(\eta, \xi)}\right)$ with $H_{4}=\left(1+i t \eta S_{\eta}(\eta, \xi)\right)^{-1}$ we integrate by parts

$$
\begin{aligned}
& \mathscr{V}^{*} \phi=C t^{1 / 2} \int_{0}^{\infty} e^{i t S(\eta, \xi)} \phi(\eta) \eta \partial_{\eta}\left(H_{4} \sqrt{\Lambda^{\prime \prime}(\eta)}\right) \\
& +e^{i t S(\eta, \xi)} \phi_{\eta}(\eta) \eta H_{4} \sqrt{\Lambda^{\prime \prime}(\eta)} d \eta .
\end{aligned}
$$

Then using formula (82), we get

$$
\begin{aligned}
& \left|\mathscr{V}^{*} \phi\right| \leq C t^{1 / 2} \int_{0}^{\infty}|\phi(\eta)|\left|\eta \partial_{\eta}\left(H_{4} \sqrt{\Lambda^{\prime \prime}(\eta)}\right)\right| \\
& +\left|\frac{\Lambda^{\prime \prime \prime}(\eta)}{\sqrt{\Lambda^{\prime \prime}(\eta)}} \eta H_{4}\right||\phi(\eta)| \\
& +\left|\left(\Lambda^{\prime \prime}(\eta)\right)^{3 / 2} \eta H_{4}\right|\left|t \mathscr{A}_{0} \phi(\eta)\right| d \eta .
\end{aligned}
$$


Then using the estimates

$$
\begin{aligned}
& \left|\eta \partial_{\eta}\left(H_{4} \sqrt{\Lambda^{\prime \prime}(\eta)}\right)\right|+\left|\frac{\Lambda^{\prime \prime \prime}(\eta)}{\sqrt{\Lambda^{\prime \prime}(\eta)}} \eta H_{4}\right| \\
& \leq \frac{C \eta^{-3 / 2}\langle\eta\rangle^{2}}{1+t \eta^{-2}\langle\eta\rangle^{4}(|\xi|+\eta)}, \\
& \left|\left(\Lambda^{\prime \prime}(\eta)\right)^{3 / 2} \eta H_{4}\right| \leq \frac{C \eta^{-7 / 2}\langle\eta\rangle^{6}}{1+t \eta^{-2}\langle\eta\rangle^{4}(|\xi|+\eta)}
\end{aligned}
$$

in the domain $\xi<0$ and $\eta>0$, we get

$$
\begin{aligned}
& \left|\mathscr{V}^{*} \phi\right| \leq C t^{1 / 2}\|\phi\|_{L^{\infty}} \int_{0}^{\infty} \frac{\eta^{-3 / 2}\langle\eta\rangle^{2} d \eta}{1+t \eta^{-2}\langle\eta\rangle^{4}(|\xi|+\eta)} \\
& +C t^{1 / 2}\left\||\eta|^{-9 / 4}\langle\eta\rangle^{4} t \mathscr{A}_{0} \phi\right\|_{L^{2}} \\
& \quad\left(\int_{0}^{\infty} \frac{\eta^{-5 / 2}\langle\eta\rangle^{6} d \eta}{\left(1+t \eta^{-2}\langle\eta\rangle^{4}(|\xi|+\eta)\right)^{2}}\right)^{1 / 2} .
\end{aligned}
$$

Therefore

$$
\begin{aligned}
& \left|\mathscr{V}^{*} \phi\right| \leq C t^{-1 / 2}\|\phi\|_{\mathbf{L}^{\infty}} \int_{0}^{\infty} \frac{\eta^{1 / 2}\langle\eta\rangle^{-2} d \eta}{|\xi|+\eta} \\
& +C t^{-1 / 2}\left\||\eta|^{-9 / 4}\langle\eta\rangle^{4} t \mathscr{A}_{0} \phi\right\|_{\mathbf{L}^{2}} \\
& \quad \cdot\left(\int_{0}^{\infty} \frac{\eta^{3 / 2}\langle\eta\rangle^{-3} d \eta}{(|\xi|+\eta)^{2}}\right)^{1 / 2} \leq C t^{-1 / 2}\langle\xi\rangle^{-1}\|\phi\|_{\mathbf{L}^{\infty}} \\
& \quad+C t^{-1 / 2}\langle\xi\rangle^{-1}\left\||\eta|^{-9 / 4}\langle\eta\rangle^{4} t \mathscr{A}_{0} \phi\right\|_{\mathbf{L}^{2}} \cdot
\end{aligned}
$$

Lemma 3 is proved.

3.3. Estimates for Derivatives. Denote $\mathscr{A}(t)=\bar{M}\left(1 / t \sqrt{\Lambda^{\prime \prime}}\right) \partial_{\eta}(1 /$ $\left.\sqrt{\Lambda^{\prime \prime}}\right) M=\mathscr{A}_{0}(t)+i \eta, \mathscr{A}_{0}(t)=\left(1 / t \sqrt{\Lambda^{\prime \prime}}\right) \partial_{\eta}\left(1 / \sqrt{\Lambda^{\prime \prime}}\right)$ such that

$$
\begin{aligned}
& i \xi \mathscr{V}^{*}(t) \phi=\frac{|t|^{1 / 2}}{\sqrt{2 \pi}} e^{i t \Lambda(\xi)} \int_{0}^{\infty} i \xi e^{-i t \Lambda^{\prime}(\eta) \xi} M(t, \eta) \phi(\eta) \\
& \cdot \sqrt{\Lambda^{\prime \prime}(\eta)} d \eta=\frac{|t|^{1 / 2}}{\sqrt{2 \pi}} \\
& \cdot e^{i t \Lambda(\xi)} \int_{0}^{\infty} e^{-i t \Lambda^{\prime}(\eta) \xi} \partial_{\eta}\left(\frac{1}{t \sqrt{\Lambda^{\prime \prime}(\eta)}} M(t, \eta)\right. \\
& \cdot \phi(\eta)) d \eta=\mathscr{V}^{*}(t) \mathscr{A}(t) \phi .
\end{aligned}
$$

Since $\left\|\mathscr{V}^{*}(t) \phi\right\|_{\mathbf{L}^{\infty}} \leq C|t|^{1 / 2}\left\|\sqrt{\Lambda^{\prime \prime}} \phi\right\|_{\mathbf{L}^{1}(0, \infty)}$ and

$$
\begin{aligned}
\left\|\mathscr{V}^{*}(t) \phi\right\|_{\mathrm{L}^{2}} & =\left\|\mathscr{F} \mathscr{U}(-t) \mathscr{D}_{t} \mathscr{B} M \phi\right\|_{\mathrm{L}^{2}} \\
& =\left\|\mathscr{D}_{t} \mathscr{B} M \phi\right\|_{\mathrm{L}^{2}}=\|\mathscr{B} M \phi\|_{\mathbf{L}^{2}}=\|\phi\|_{\mathrm{L}^{2}},
\end{aligned}
$$

then by the Riesz interpolation theorem (see [23], p. 52) we have

$$
\left\|\mathscr{V}^{*}(t) \phi\right\|_{L^{p}} \leq C|t|^{1 / 2-1 / p}\left\|\left(\Lambda^{\prime \prime}\right)^{1 / 2-1 / p} \phi\right\|_{\mathbf{L}^{p /(p-1)}}
$$

for $2 \leq p \leq \infty$. We now estimate the derivative $\partial_{\eta} \mathscr{V} \phi$.

Lemma 4. The estimate

$$
\left\|\eta^{\gamma+j}\langle\eta\rangle^{-2 \gamma} t \mathscr{A}_{0} \mathscr{V} \xi^{j} \phi\right\|_{\mathbf{L}^{2}} \leq C\left\|\xi \phi_{\xi}\right\|_{\mathbf{L}^{2}}+\left\||\xi|^{1 / 2} \phi\right\|_{\mathbf{L}^{\infty}}
$$

is true for all $t \geq 1, j=-1,0$, where $\gamma>0$.

Proof. Since $\mathscr{A}_{0}=\mathscr{A}-i \eta$ and $\mathscr{A} \mathscr{V}=\mathscr{V} i \xi$, we have

$$
\begin{aligned}
& t \mathscr{A}_{0} \mathscr{V} \xi^{j} \phi=i t\left(\mathscr{V} \xi^{j+1} \phi-\eta \mathscr{V} \xi^{j} \phi\right) \\
& \quad=\sqrt{\frac{t \Lambda^{\prime \prime}(\eta)}{2 \pi}} \int_{0}^{\infty} e^{-i t S(\eta, \xi)} i t(\xi-\eta) \xi^{j} \phi(\xi) d \xi
\end{aligned}
$$

for $\eta>0$. So we need to estimate

$$
\begin{aligned}
& \eta^{\gamma+j}\langle\eta\rangle^{-2 \gamma} t \mathscr{A}_{0} \mathscr{V} \xi^{j} \phi=\eta^{\gamma}\langle\eta\rangle^{-2 \gamma} \mathscr{V} \psi_{1}\left(\xi, \xi_{1}\right) \xi \phi_{\xi} \\
& +\eta^{\gamma}\langle\eta\rangle^{-2 \gamma} \\
& \cdot \sqrt{\frac{t \Lambda^{\prime \prime}(\eta)}{2 \pi}} \int_{0}^{\infty} e^{-i t S(\eta, \xi)}\left(\psi_{1}(\xi, \eta)-\psi_{1}\left(\xi, \xi_{1}\right)\right) \\
& \cdot \xi \phi_{\xi}(\xi) d \xi+\eta^{\gamma}\langle\eta\rangle^{-2 \gamma} \\
& \cdot \sqrt{\frac{t \Lambda^{\prime \prime}(\eta)}{2 \pi}} \int_{0}^{\infty} e^{-i t S(\eta, \xi)} \phi(\xi) \psi_{2}(\xi, \eta) d \xi=I_{1} \\
& +I_{2}+I_{3},
\end{aligned}
$$

where $\xi_{1}=(a / b)^{1 / 4}$, and

$$
\begin{aligned}
& \psi_{1}(\xi, \eta)=\frac{\eta^{2+j} \xi^{j+1}}{\left(a+b \eta^{2} \xi^{2}\right)(\xi+\eta)}, \\
& \psi_{2}(\xi, \eta)=\partial_{\xi} \frac{\eta^{2+j} \xi^{j+2}}{\left(a+b \eta^{2} \xi^{2}\right)(\xi+\eta)} .
\end{aligned}
$$

For the first summand using $\|\mathscr{V} \phi\|_{L^{2}} \leq\|\phi\|_{L^{2}}$ we have for $j=-1,0$

$$
\begin{aligned}
\left\|I_{1}\right\|_{\mathbf{L}^{2}} & =\left\|\mathscr{V} \psi_{1}\left(\xi, \xi_{1}\right) \xi \phi_{\xi}\right\|_{\mathbf{L}^{2}} \leq\left\|\langle\xi\rangle^{-3} \xi^{j+2} \phi_{\xi}\right\|_{\mathbf{L}^{2}} \\
& \leq\left\|\xi \phi_{\xi}\right\|_{\mathbf{L}^{2}} .
\end{aligned}
$$


Consider the second summand

$$
\begin{aligned}
& \left\|I_{2}\right\|_{L^{2}(0, \infty)}^{2}=C t \int_{0}^{\infty} d \eta \Lambda^{\prime \prime}(\eta) \eta^{2 \gamma}\langle\eta\rangle^{-4 \gamma} \\
& \cdot \int_{0}^{\infty} e^{-i t S(\eta, \xi)}\left(\psi_{1}(\xi, \eta)-\psi_{1}\left(\xi, \xi_{1}\right)\right) \xi \phi_{\xi}(\xi) d \xi \\
& \cdot \int_{0}^{\infty} e^{i t S(\eta, \zeta)}\left(\psi_{1}(\zeta, \eta)-\psi_{1}\left(\zeta, \xi_{1}\right)\right) \zeta \overline{\phi_{\zeta}(\zeta)} d \zeta \\
& =C \int_{0}^{\infty} d \xi e^{-i t \Lambda(\xi)} \xi \phi_{\xi}(\xi) \int_{0}^{\infty} d \zeta \\
& \cdot e^{i t \Lambda(\zeta)} \overline{\zeta \phi_{\zeta}(\zeta)} K(t, \xi, \zeta),
\end{aligned}
$$

$$
\begin{aligned}
& \cdot\left(\psi_{1}(\xi, \eta(x))-\psi_{1}\left(\xi, \xi_{1}\right)\right) \\
& \cdot\left(\psi_{1}(\zeta, \eta(x))-\psi_{1}\left(\zeta, \xi_{1}\right)\right) .
\end{aligned}
$$

We can rotate the contour of integration $x=r e^{i(\pi / 8) \operatorname{sgn}(\xi-\zeta)}$, since we see that $\eta(x)=C x^{1 / 2}$ for $x \rightarrow+\infty, \eta(x)=\eta(0)=$ $(a / b)^{1 / 4}$ for $x \rightarrow 0$, and $\eta(x)=C|x|^{-1 / 2}$ for $x \rightarrow-\infty$, and hence

$|K(t, \xi, \zeta)|$

$$
\leq C t \int_{-\infty}^{\infty} e^{-C t|\xi-\zeta| r \mid} \mid \psi_{1}\left(\xi, \eta\left(r e^{i(\pi / 8) \operatorname{sgn}(\xi-\zeta)}\right)\right)
$$

$$
\begin{aligned}
& -\psi_{1}(\xi, \eta(0))|| \psi_{1}\left(\zeta, \eta\left(r e^{i(\pi / 8) \operatorname{sgn}(\xi-\zeta)}\right)\right) \\
& -\psi_{1}(\zeta, \eta(0)) \mid \eta^{2 \gamma}\langle\eta\rangle^{-4 \gamma} d r \\
& \leq C t \int_{1}^{\infty} e^{-C t|\xi-\zeta||r|}\langle r\rangle^{-2 \gamma} d r \\
& +C t \int_{-1}^{1} e^{-C t|\xi-\zeta||r|}|r| d r \\
& +C t \int_{-\infty}^{-1} e^{-C t|\xi-\zeta||r|}|r|^{-\gamma} d r \leq C t(|\xi-\zeta| t)^{\gamma-1} \\
& \cdot\langle(\xi-\zeta) t\rangle^{-2 \gamma} .
\end{aligned}
$$

Then by the Young inequality we obtain

$$
\begin{gathered}
\left\|I_{2}\right\|_{\mathbf{L}^{2}(0, \infty)}^{2} \leq C t\left\|\xi \phi_{\xi}\right\|_{\mathbf{L}^{2}} \\
\cdot\left\|\int_{\mathbf{R}}(|\xi-\zeta| t)^{\gamma-1}\langle(\xi-\zeta) t\rangle^{-2 \gamma}\left|\zeta \phi_{\zeta}(\zeta)\right| d \zeta\right\|_{L^{2}} \\
\quad \leq C t\left\|\xi \phi_{\xi}\right\|_{\mathbf{L}^{2}}^{2}\left\|(\xi t)^{\gamma-1}\langle\xi t\rangle^{-2 \gamma}\right\|_{\mathbf{L}^{1}} \leq C\left\|\xi \phi_{\xi}\right\|_{\mathbf{L}^{2}}^{2} .
\end{gathered}
$$

To estimate $I_{3}$ we integrate by parts via identity (47)

$$
I_{3}=C t^{1 / 2} \eta^{\gamma-3 / 2}\langle\eta\rangle^{2-2 \gamma}
$$

$$
\begin{aligned}
& \cdot \int_{0}^{\infty} e^{-i t S(\eta, \xi)}(\xi-\eta) H_{1} \psi_{2}(\xi, \eta) \phi_{\xi}(\xi) d \xi \\
& +C t^{1 / 2} \eta^{\gamma-3 / 2}\langle\eta\rangle^{2-2 \gamma} \\
& \cdot \int_{0}^{\infty} e^{-i t S(\eta, \xi)} \phi(\xi)(\xi-\eta) \partial_{\xi}\left(H_{1} \psi_{2}(\xi, \eta)\right) d \xi .
\end{aligned}
$$

Using the estimates

$$
\begin{aligned}
& \left|(\xi-\eta) H_{1} \psi_{2}(\xi, \eta)\right| \\
& \leq \frac{C|\xi-\eta| \eta^{2+j} \xi^{j+1}}{\left(1+t(\xi-\eta)^{2} \xi^{-2} \eta^{-2}\left(1+\xi^{2} \eta^{2}\right)(\xi+\eta)\right)\left(1+\xi^{2} \eta^{2}\right)(\xi+\eta)}, \\
& \left|(\xi-\eta) \partial_{\xi}\left(H_{1} \psi_{2}(\xi, \eta)\right)\right| \\
& \leq \frac{C \eta^{2+j}\left(|\xi-\eta| \xi^{j}+\xi^{j+1}\right)}{\left(1+t(\xi-\eta)^{2} \xi^{-2} \eta^{-2}\left(1+\xi^{2} \eta^{2}\right)(\xi+\eta)\right)\left(1+\xi^{2} \eta^{2}\right)(\xi+\eta)}
\end{aligned}
$$

we obtain

$$
\begin{aligned}
\left|I_{3}\right| \leq C t^{1 / 2} \int_{0}^{\infty} \frac{\eta^{\gamma-3 / 2}\langle\eta\rangle^{2-2 \gamma}|\xi-\eta| \eta^{2+j} \xi^{j}\left|\xi \phi_{\xi}(\xi)\right| d \xi}{\left(1+t(\xi-\eta)^{2} \xi^{-2} \eta^{-2}\left(1+\xi^{2} \eta^{2}\right)(\xi+\eta)\right)\left(1+\xi^{2} \eta^{2}\right)(\xi+\eta)} \\
+C t^{1 / 2} \int_{0}^{\infty} \frac{\eta^{\gamma-3 / 2}\langle\eta\rangle^{2-2 \gamma} \eta^{2+j}\left(|\xi-\eta| \xi^{j}+\xi^{j+1}\right)|\phi(\xi)| d \xi}{\left(1+t(\xi-\eta)^{2} \xi^{-2} \eta^{-2}\left(1+\xi^{2} \eta^{2}\right)(\xi+\eta)\right)\left(1+\xi^{2} \eta^{2}\right)(\xi+\eta)}
\end{aligned}
$$




$$
\begin{aligned}
\leq & C t^{1 / 2}\left\|\xi \phi_{\xi}\right\|_{L^{2}}\left(\int_{0}^{\infty} \frac{\eta^{2 \gamma+2 j+1}\langle\eta\rangle^{4-4 \gamma}|\xi-\eta|^{2} \xi^{2 j} d \xi}{\left(1+t(\xi-\eta)^{2} \xi^{-2} \eta^{-2}\left(1+\xi^{2} \eta^{2}\right)(\xi+\eta)\right)^{2}\left(1+\xi^{2} \eta^{2}\right)^{2}(\xi+\eta)^{2}}\right)^{1 / 2} \\
& +C t^{1 / 2}\left\||\xi|^{1 / 2} \phi\right\|_{L^{\infty}} \int_{0}^{\infty} \frac{\eta^{\gamma-3 / 2}\langle\eta\rangle^{2-2 \gamma} \eta^{2+j}\left(|\xi-\eta| \xi^{j}+\xi^{j+1}\right) \xi^{-1 / 2} d \xi}{\left(1+t(\xi-\eta)^{2} \xi^{-2} \eta^{-2}\left(1+\xi^{2} \eta^{2}\right)(\xi+\eta)\right)\left(1+\xi^{2} \eta^{2}\right)(\xi+\eta)} .
\end{aligned}
$$

\section{Since changing $\xi=\eta y$}

$$
\begin{aligned}
& \int_{0}^{\infty} \frac{t \eta^{2 \gamma+2 j+1}\langle\eta\rangle^{4-4 \gamma}|\xi-\eta|^{2} \xi^{2 j} d \xi}{\left(1+t(\xi-\eta)^{2} \xi^{-2} \eta^{-2}\left(1+\xi^{2} \eta^{2}\right)(\xi+\eta)\right)^{2}\left(1+\xi^{2} \eta^{2}\right)^{2}(\xi+\eta)^{2}} \\
& =\int_{0}^{\infty} \frac{t \eta^{2 \gamma+4 j+2}\langle\eta\rangle^{4-4 \gamma}|y-1|^{2} y^{2 j} d y}{\left(1+t(y-1)^{2} y^{-2} \eta^{-1}\left(1+y^{2} \eta^{4}\right)(y+1)\right)^{2}\left(1+y^{2} \eta^{4}\right)^{2}(y+1)^{2}} \leq C t^{-1} \eta^{2 \gamma+4 j+4}\langle\eta\rangle^{4-4 \gamma} \int_{0}^{1 / 2} \frac{y^{2 j+4} d y}{\left(1+y \eta^{2}\right)^{8}} \\
& +C t \eta^{2 \gamma+4 j+2}\langle\eta\rangle^{-4-4 \gamma} \int_{1 / 2}^{3 / 2} \frac{|y-1|^{2} d y}{\left(1+t \eta^{-1}\langle\eta\rangle^{4}(y-1)^{2}\right)^{2}}+C t^{-1} \eta^{2 \gamma+4 j+4}\langle\eta\rangle^{4-4 \gamma} \int_{3 / 2}^{\infty} \frac{y^{2 j-2} d y}{\left(1+y \eta^{2}\right)^{8}} \leq C \eta^{2 \gamma-1}\langle\eta\rangle^{-4 \gamma} \\
& t^{1 / 2} \int_{0}^{\infty} \frac{\eta^{\gamma-3 / 2}\langle\eta\rangle^{2-2 \gamma} \eta^{2+j}\left(|\xi-\eta| \xi^{j}+\xi^{j+1}\right) \xi^{-1 / 2} d \xi}{\left(1+t(\xi-\eta)^{2} \xi^{-2} \eta^{-2}\left(1+\xi^{2} \eta^{2}\right)(\xi+\eta)\right)\left(1+\xi^{2} \eta^{2}\right)(\xi+\eta)} \\
& =t^{1 / 2} \int_{0}^{\infty} \frac{\eta^{2 j+1+\gamma}\langle\eta\rangle^{2-2 \gamma}(|y-1|+y) y^{j-1 / 2} d y}{\left(1+t(y-1)^{2} y^{-2} \eta^{-1}\left(1+y^{2} \eta^{4}\right)(y+1)\right)\left(1+y^{2} \eta^{4}\right)(y+1)} \leq C t^{-1 / 2} \eta^{2 j+2+\gamma}\langle\eta\rangle^{2-2 \gamma} \int_{0}^{1 / 2} \frac{y^{j+3 / 2} d y}{\left(1+y \eta^{2}\right)^{4}} \\
& +C t^{1 / 2} \eta^{2 j+1+\gamma}\langle\eta\rangle^{-2-2 \gamma} \int_{1 / 2}^{3 / 2} \frac{d y}{1+t \eta^{-1}\langle\eta\rangle^{4}(y-1)^{2}}+C t^{-1 / 2} \eta^{2 j+2+\gamma}\langle\eta\rangle^{2-2 \gamma} \int_{3 / 2}^{\infty} \frac{y^{j-3 / 2} d y}{\left(1+y \eta^{2}\right)^{4}} \leq C \eta^{\gamma-1 / 2}\langle\eta\rangle^{-2 \gamma},
\end{aligned}
$$

we get

$\left\|I_{3}\right\|_{\mathbf{L}^{2}(0, \infty)}$

$$
\begin{aligned}
& \leq C\left(\left\|\xi \phi_{\xi}\right\|_{\mathbf{L}^{2}}+\left\||\xi|^{1 / 2} \phi\right\|_{\mathbf{L}^{\infty}}\right)\left\|\eta^{\gamma-1 / 2}\langle\eta\rangle^{-2 \gamma}\right\|_{\mathbf{L}^{2}(0, \infty)} \\
& \leq C\left\|\xi \phi_{\xi}\right\|_{\mathbf{L}^{2}}+C\left\||\xi|^{1 / 2} \phi\right\|_{\mathbf{L}^{\infty}} .
\end{aligned}
$$

In the case of $\eta<0$, the same estimate is obtained easier than the case of the positive line. Lemma 4 is proved.

We need estimate of $\mathscr{V}_{t}$.

Lemma 5. The estimate

$$
\left\|\eta^{\gamma}\langle\eta\rangle^{-2 \gamma-2} t \partial_{t} \mathscr{V} \phi\right\|_{\mathbf{L}^{2}} \leq C\left\|\xi \phi_{\xi}\right\|_{\mathbf{L}^{2}}+C\left\||\xi|^{1 / 2} \phi\right\|_{\mathbf{L}^{\infty}}
$$

is true for all $t \geq 1$, where $\gamma>0$.

Proof. Since

$$
\frac{S(\eta, \xi)}{\partial_{\xi} S(\eta, \xi)}=\frac{\left(3 a+2 b \eta^{3} \xi+b \eta^{2} \xi^{2}\right)(\xi-\eta) \xi}{3\left(a+b \eta^{2} \xi^{2}\right)(\xi+\eta)}
$$

integrating by parts we get with $\xi_{1}=(a / b)^{1 / 4}$

$$
\begin{aligned}
& \eta^{\gamma}\langle\eta\rangle^{-2 \gamma-2} t \mathscr{V}_{t} \phi=\eta^{\gamma}\langle\eta\rangle^{-2 \gamma-2} \frac{1}{2} \mathscr{V} \phi \\
& -i t \eta^{\gamma}\langle\eta\rangle^{-2 \gamma-2} \sqrt{\frac{t \Lambda^{\prime \prime}(\eta)}{2 \pi}} \int_{0}^{\infty} e^{-i t S(\eta, \xi)} S(\eta, \xi) \\
& \cdot \phi(\xi) d \xi=\frac{1}{2} \eta^{\gamma}\langle\eta\rangle^{-2 \gamma-2} \mathscr{V} \phi-\eta^{\gamma}\langle\eta\rangle^{-2 \gamma-1} \\
& \cdot \mathscr{V}\left(\psi_{1}(\xi, \xi) \xi \phi_{\xi}\right)-\eta^{\gamma}\langle\eta\rangle^{-2 \gamma-2} \\
& \cdot \sqrt{\frac{t \Lambda^{\prime \prime}(\eta)}{2 \pi}} \int_{0}^{\infty} e^{-i t S(\eta, \xi)}\left(\psi_{1}(\xi, \eta)-\psi_{1}(\xi, \xi 1)\right) \\
& \cdot \xi \phi_{\xi}(\xi) d \xi-\eta^{\gamma}\langle\eta\rangle^{-2 \gamma-2} \\
& \cdot \sqrt{\frac{t \Lambda^{\prime \prime}(\eta)}{2 \pi}} \int_{0}^{\infty} e^{-i t S(\eta, \xi)} \psi_{2}(\xi, \eta) \phi(\xi) d \xi
\end{aligned}
$$




$$
\begin{aligned}
& -\eta^{\gamma}\langle\eta\rangle^{-2 \gamma-2} \sqrt{\frac{t \Lambda^{\prime \prime}(\eta)}{2 \pi}} \int_{0}^{\infty} e^{-i t S(\eta, \xi)} \psi_{3}(\xi, \eta) \\
& \cdot \phi(\xi) d \xi=I_{1}+I_{2}+I_{3}+I_{4}+I_{5},
\end{aligned}
$$

where

$$
\begin{aligned}
& \psi_{1}(\xi, \eta)=\frac{\left(3 a+2 b \eta^{3} \xi+b \eta^{2} \xi^{2}\right)(\xi-\eta)}{3\left(a+b \eta^{2} \xi^{2}\right)(\xi+\eta)}, \\
& \psi_{2}(\xi, \eta)=(\xi-\eta) \partial_{\xi} \frac{\left(3 a+2 b \eta^{3} \xi+b \eta^{2} \xi^{2}\right) \xi}{3\left(a+b \eta^{2} \xi^{2}\right)(\xi+\eta)} \\
& \psi_{3}(\xi, \eta)=\frac{\left(3 a+2 b \eta^{3} \xi+b \eta^{2} \xi^{2}\right) \xi}{3\left(a+b \eta^{2} \xi^{2}\right)(\xi+\eta)} .
\end{aligned}
$$

Using the estimate $\|\mathscr{V} \phi\|_{L^{2}} \leq\|\phi\|_{\mathbf{L}^{2}}$ we find for the first summand

$$
\left\|I_{1}\right\|_{\mathbf{L}^{2}} \leq\|\mathscr{V} \phi\|_{\mathbf{L}^{2}} \leq\|\phi\|_{\mathbf{L}^{2}}
$$

and for the second summand

$$
\begin{aligned}
\left\|I_{2}\right\|_{\mathbf{L}^{2}(0, \infty)} & \leq\left\|\eta^{\gamma}\langle\eta\rangle^{-2 \gamma-2} \mathscr{V}\left(\psi_{1}\left(\xi, \xi_{1}\right) \xi \phi_{\xi}\right)\right\|_{\mathbf{L}^{2}(0, \infty)} \\
& \leq\left\|\psi_{1}\left(\xi, \xi_{1}\right) \xi \phi_{\xi}\right\|_{\mathbf{L}^{2}(0, \infty)} \leq C\left\|\xi \phi_{\xi}\right\|_{\mathbf{L}^{2}} .
\end{aligned}
$$

Consider the third summand

$$
\begin{aligned}
& \left\|\eta^{\gamma}\langle\eta\rangle^{-2 \gamma-2} I_{3}\right\|_{L^{2}(0, \infty)}^{2}=C t \int_{0}^{\infty} d \eta \Lambda^{\prime \prime}(\eta) \\
& \cdot \eta^{2 \gamma}\langle\eta\rangle^{-4 \gamma-4} \\
& \cdot \int_{0}^{\infty} e^{-i t S(\eta, \xi)}\left(\psi_{1}(\xi, \eta)-\psi_{1}\left(\xi, \xi_{1}\right)\right) \xi \phi_{\xi}(\xi) d \xi \\
& \cdot \int_{0}^{\infty} e^{i t S(\eta, \zeta)}\left(\psi_{1}(\xi, \eta)-\psi_{1}\left(\xi, \xi_{1}\right)\right) \zeta \overline{\phi_{\zeta}(\zeta)} d \zeta \\
& =C \int_{0}^{\infty} d \xi e^{-i t \Lambda(\xi)} \xi \phi_{\xi}(\xi) \int_{0}^{\infty} d \zeta \\
& \cdot e^{i t \Lambda(\zeta)} \overline{\zeta \phi_{\zeta}(\zeta)} K_{1}(t, \xi, \zeta),
\end{aligned}
$$

where

$$
\begin{aligned}
K_{1}(t, \xi, \zeta)= & t \int_{0}^{\infty} d \eta \Lambda^{\prime \prime}(\eta) \eta^{2 \gamma}\langle\eta\rangle^{-4 \gamma-4} \\
& \cdot e^{i t \Lambda^{\prime}(\eta)(\xi-\zeta)}\left(\psi_{1}(\xi, \eta)-\psi_{1}\left(\xi, \xi_{1}\right)\right) \\
& \cdot\left(\psi_{1}(\zeta, \eta)-\psi_{1}\left(\zeta, \xi_{1}\right)\right) .
\end{aligned}
$$

Changing $x=\Lambda^{\prime}(\eta), \eta(x)=\sqrt{(1 / 2 b)\left(x+\sqrt{4 a b+x^{2}}\right)}$, we get

$$
\begin{gathered}
K_{1}(t, \xi, \zeta)=t \int_{-\infty}^{\infty} d x \eta^{2 \gamma}(x)\langle\eta(x)\rangle^{-4 \gamma-4} \\
\cdot e^{i t x(\xi-\zeta)}\left(\psi_{1}(\xi, \eta(x))-\psi_{1}(\xi, \eta(0))\right) \\
\cdot\left(\psi_{1}(\zeta, \eta(x))-\psi_{1}(\zeta, \eta(0))\right) .
\end{gathered}
$$

We can rotate the contour of integration $x=r e^{i(\pi / 8) \operatorname{sgn}(\xi-\zeta)}$, since we see that $\eta(x)=C x^{1 / 2}$ for $x \rightarrow+\infty, \eta(x)=\eta(0)=\xi_{1}$ for $x \rightarrow 0$, and $\eta(x)=C|x|^{-1 / 2}$ for $x \rightarrow-\infty$, and hence

$$
\begin{aligned}
& \left|K_{1}(t, \xi, \zeta)\right| \\
& \quad \leq C t \int_{-\infty}^{\infty} e^{-C t|\xi-\zeta||r|} \psi_{1}\left(\psi_{1}\left(\xi, \eta\left(r e^{i(\pi / 8) \operatorname{sgn}(\xi-\zeta)}\right)\right)\right. \\
& \left.\quad-\psi_{1}(\xi, \eta(0))\right)\left(\psi_{1}\left(\zeta, \eta\left(r e^{i(\pi / 8) \operatorname{sgn}(\xi-\zeta)}\right)\right)\right. \\
& \left.\quad-\psi_{1}(\zeta, \eta(0))\right) \eta^{2 \gamma}\langle\eta\rangle^{-4 \gamma-4} d r \\
& \quad \leq C t \int_{1}^{\infty} e^{-C t|\xi-\zeta||r|}\langle r\rangle^{-\gamma} d r \\
& \quad+C t \int_{-1}^{1} e^{-C t|\xi-\zeta||r|}|r| d r \\
& \quad+C t \int_{-\infty}^{-1} e^{-C t|\xi-\zeta||r|}|r|^{-2 \gamma} d r \leq C t(|\xi-\zeta| t)^{\gamma-1} \\
& \quad \cdot\langle(\xi-\zeta) t\rangle^{-2 \gamma} .
\end{aligned}
$$

Then by the Young inequality we obtain

$$
\begin{aligned}
& \left\|I_{3}\right\|_{\mathbf{L}^{2}(0, \infty)}^{2} \leq C t\left\|\xi \phi_{\xi}\right\|_{\mathbf{L}^{2}} \\
& \cdot\left\|\int_{\mathbf{R}}(|\xi-\zeta| t)^{\gamma-1}\langle(\xi-\zeta) t\rangle^{-2 \gamma}\left|\zeta \phi_{\zeta}(\zeta)\right| d \zeta\right\|_{\mathbf{L}^{2}} \\
& \quad \leq C t\left\|\xi \phi_{\xi}\right\|_{\mathbf{L}^{2}}^{2}\left\|(\xi t)^{\gamma-1}\langle\xi t\rangle^{-2 \gamma}\right\|_{\mathbf{L}^{1}} \leq C\left\|\xi \phi_{\xi}\right\|_{\mathbf{L}^{2}}^{2} .
\end{aligned}
$$

Next we estimate $I_{4}$. We integrate by parts via identity (53) to get

$$
\begin{aligned}
I_{4} & =C t^{1 / 2} \eta^{\gamma-3 / 2}\langle\eta\rangle^{-2 \gamma} \\
& \cdot \int_{0}^{\infty} e^{-i t S(\eta, \xi)} \phi(\xi) \xi \partial_{\xi}\left(H_{2} \psi_{2}(\xi, \eta)\right) \\
& +e^{-i t S(x, \xi)} H_{2} \psi_{2}(\xi, \eta) \xi \phi_{\xi}(\xi) d \xi .
\end{aligned}
$$

Using the estimates $\left|\psi_{2}(\xi, \eta)\right| \leq C\left(|\xi-\eta|\langle\eta\rangle^{2} /(\xi+\eta)\right)$,

$$
\begin{aligned}
& \left|H_{2} \psi_{2}(\xi, \eta)\right| \\
& \leq \frac{C|\xi-\eta|\langle\eta\rangle^{2}}{\left(1+t \xi^{-1} \eta^{-2}\left(1+\eta^{2} \xi^{2}\right)\left|\xi^{2}-\eta^{2}\right|\right)(\xi+\eta)}, \\
& \left|\xi \partial_{\xi}\left(H_{2} \psi_{2}\right)\right| \leq \frac{C\langle\eta\rangle^{2}}{1+t \xi^{-1} \eta^{-2}\left(1+\eta^{2} \xi^{2}\right)\left|\xi^{2}-\eta^{2}\right|}
\end{aligned}
$$


we obtain

$$
\begin{aligned}
& \left|I_{4}\right| \leq C t^{1 / 2} \eta^{\gamma-3 / 2}\langle\eta\rangle^{2-2 \gamma} \\
& \quad \cdot \int_{0}^{\infty} \frac{|\phi(\xi)| d \xi}{1+t \xi^{-1} \eta^{-2}\left(1+\eta^{2} \xi^{2}\right)\left|\xi^{2}-\eta^{2}\right|} \\
& \quad+C t^{1 / 2} \eta^{\gamma-3 / 2}\langle\eta\rangle^{2-2 \gamma} \\
& \quad \cdot \int_{0}^{\infty} \frac{|\xi-\eta|\left|\xi \phi_{\xi}(\xi)\right| d \xi}{\left(1+t \xi^{-1} \eta^{-2}\left(1+\eta^{2} \xi^{2}\right)\left|\xi^{2}-\eta^{2}\right|\right)(\xi+\eta)} \\
& \quad \leq C\left\||\xi|^{1 / 2} \phi\right\|_{L^{\infty}} \int_{0}^{\infty} \frac{t^{1 / 2} \eta^{\gamma-3 / 2}\langle\eta\rangle^{2-2 \gamma} \xi^{-1 / 2} d \xi}{1+t \xi^{-1} \eta^{-2}\left(1+\eta^{2} \xi^{2}\right)\left|\xi^{2}-\eta^{2}\right|} \\
& \quad+C\left\|\xi \phi_{\xi}\right\|_{L^{2}} \\
& \quad \cdot\left(\int_{0}^{\infty} \frac{t \eta^{2 \gamma-3}\langle\eta\rangle^{4-4 \gamma}(\xi-\eta)^{2} d \xi}{\left(1+t \xi^{-1} \eta^{-2}\left(1+\eta^{2} \xi^{2}\right)\left|\xi^{2}-\eta^{2}\right|\right)^{2}(\xi+\eta)^{2}}\right)^{1 / 2} .
\end{aligned}
$$

Changing $\xi=\eta y$ we find

$$
\begin{aligned}
& \int_{0}^{\infty} \frac{t^{1 / 2} \eta^{\gamma-3 / 2}\langle\eta\rangle^{2-2 \gamma} \xi^{-1 / 2} d \xi}{1+t \xi^{-1} \eta^{-2}\left(1+\eta^{2} \xi^{2}\right)\left|\xi^{2}-\eta^{2}\right|} \\
& =\int_{0}^{\infty} \frac{t^{1 / 2} \eta^{\gamma-1}\langle\eta\rangle^{2-2 \gamma} y^{-1 / 2} d y}{1+t \eta^{-1} y^{-1}\left(1+\eta^{4} y^{2}\right)(y+1)|y-1|} \\
& \leq C t^{-1 / 2} \eta^{\gamma}\langle\eta\rangle^{2-2 \gamma} \int_{0}^{1 / 2} \frac{y^{1 / 2} d y}{\left(1+\eta^{2} y\right)^{2}} \\
& \quad+C t^{1 / 2} \eta^{\gamma-1}\langle\eta\rangle^{2-2 \gamma} \int_{1 / 2}^{3 / 2} \frac{d y}{1+t \eta^{-1}\langle\eta\rangle^{4}|y-1|} \\
& \quad+C t^{-1 / 2} \eta^{\gamma}\langle\eta\rangle^{2-2 \gamma} \int_{3 / 2}^{\infty} \frac{y^{-3 / 2} d y}{1+\eta^{4} y^{2}} \leq C \eta^{\gamma-1 / 2}\langle\eta\rangle^{-2 \gamma} \\
& \int_{0}^{\infty} \frac{t \eta^{2 \gamma-3}\langle\eta\rangle^{4-4 \gamma}(\xi-\eta)^{2} d \xi}{\left(1+t \xi^{-1} \eta^{-2}\left(1+\eta^{2} \xi^{2}\right)\left|\xi^{2}-\eta^{2}\right|\right)^{2}(\xi+\eta)^{2}} \\
& =\int_{0}^{\infty} \frac{t \eta^{2 \gamma-2}\langle\eta\rangle^{4-4 \gamma}(y-1)^{2} d y}{\left(1+t \eta^{-1} y^{-1}\left(1+\eta^{4} y^{2}\right)(y+1)|y-1|\right)^{2}(y+1)^{2}}
\end{aligned}
$$

$$
\begin{aligned}
& \leq C t^{-1} \eta^{2 \gamma}\langle\eta\rangle^{4-4 \gamma} \int_{0}^{1 / 2} \frac{y^{2} d y}{\left(1+\eta^{2} y\right)^{4}} \\
&+C t^{-1} \eta^{2 \gamma}\langle\eta\rangle^{-4-4 \gamma} \int_{1 / 2}^{3 / 2} d y \\
&+C t^{-1} \eta^{2 \gamma}\langle\eta\rangle^{4-4 \gamma} \int_{3 / 2}^{\infty} \frac{d y}{y^{2}\left(1+\eta^{2} y\right)^{4}} \\
& \leq C \eta^{2 \gamma-1}\langle\eta\rangle^{-4 \gamma} .
\end{aligned}
$$

Hence we get

$\left\|I_{4}\right\|_{\mathbf{L}^{2}(0, \infty)}$

$$
\begin{aligned}
& \leq C\left(\left\|\xi \phi_{\xi}\right\|_{\mathbf{L}^{2}}+\left\||\xi|^{1 / 2} \phi\right\|_{\mathbf{L}^{\infty}}\right)\left\|\eta^{\gamma-1 / 2}\langle\eta\rangle^{-2 \gamma}\right\|_{\mathbf{L}^{2}(0, \infty)} \\
& \leq C\left\|\xi \phi_{\xi}\right\|_{\mathbf{L}^{2}(0, \infty)}+C\left\||\xi|^{1 / 2} \phi\right\|_{\mathbf{L}^{\infty}} .
\end{aligned}
$$

In the last integral $I_{5}$ we integrate by parts via identity (47)

$$
\begin{aligned}
I_{5} & =C t^{1 / 2} \eta^{\gamma-3 / 2}\langle\eta\rangle^{-2 \gamma} \\
& \cdot \int_{0}^{\infty} e^{-i t S(\eta, \xi)}(\xi-\eta) H_{1} \psi_{3}(\xi, \eta) \phi_{\xi}(\xi) \\
& +e^{-i t S(\eta, \xi)} \phi(\xi)(\xi-\eta) \partial_{\xi}\left(H_{1} \psi_{3}(\xi, \eta)\right) d \xi .
\end{aligned}
$$

Using the estimates $\left|\psi_{3}(\xi, \eta)\right| \leq C\left(\xi\langle\eta\rangle^{2} /(\xi+\eta)\right)$,

$$
\begin{aligned}
& \left|(\xi-\eta) H_{1} \psi_{3}(\xi, \eta)\right| \\
& \leq \frac{C \xi|\xi-\eta|\langle\eta\rangle^{2}}{\left(1+t(\xi-\eta)^{2} \xi^{-2} \eta^{-2}\left(1+\xi^{2} \eta^{2}\right)(\xi+\eta)\right)(\xi+\eta)} \\
& \left|(\xi-\eta) \partial_{\xi}\left(H_{1} \psi_{3}(\xi, \eta)\right)\right| \\
& \leq \frac{C\langle\eta\rangle^{2}}{1+t(\xi-\eta)^{2} \xi^{-2} \eta^{-2}\left(1+\xi^{2} \eta^{2}\right)(\xi+\eta)}
\end{aligned}
$$

we obtain

$$
\begin{aligned}
\left|I_{5}\right| \leq & C \int_{0}^{\infty} \frac{t^{1 / 2} \eta^{\gamma-3 / 2}\langle\eta\rangle^{2-2 \gamma}|\xi-\eta|\left|\xi \phi_{\xi}(\xi)\right| d \xi}{\left(1+t(\xi-\eta)^{2} \xi^{-2} \eta^{-2}\left(1+\xi^{2} \eta^{2}\right)(\xi+\eta)\right)(\xi+\eta)}+C \int_{0}^{\infty} \frac{t^{1 / 2} \eta^{\gamma-3 / 2}\langle\eta\rangle^{2-2 \gamma}|\phi(\xi)| d \xi}{1+t(\xi-\eta)^{2} \xi^{-2} \eta^{-2}\left(1+\xi^{2} \eta^{2}\right)(\xi+\eta)} \\
\leq & C\left\|\xi \phi_{\xi}\right\|_{L^{2}}\left(\int_{0}^{\infty} \frac{t \eta^{2 \gamma-3}\langle\eta\rangle^{4-4 \gamma}(\xi-\eta)^{2} d \xi}{\left(1+t(\xi-\eta)^{2} \xi^{-2} \eta^{-2}\left(1+\xi^{2} \eta^{2}\right)(\xi+\eta)\right)^{2}(\xi+\eta)^{2}}\right)^{1 / 2} \\
& +C\left\||\xi|^{1 / 2} \phi\right\|_{L^{\infty}} \int_{0}^{\infty} \frac{t^{1 / 2} \eta^{\gamma-3 / 2}\langle\eta\rangle^{2-2 \gamma} \xi^{-1 / 2} d \xi}{1+t(\xi-\eta)^{2} \xi^{-2} \eta^{-2}\left(1+\xi^{2} \eta^{2}\right)(\xi+\eta)} .
\end{aligned}
$$


Since changing $\xi=\eta y$

$$
\begin{aligned}
& \int_{0}^{\infty} \frac{t \eta^{2 \gamma-3}\langle\eta\rangle^{4-4 \gamma}(\xi-\eta)^{2} d \xi}{\left(1+t(\xi-\eta)^{2} \xi^{-2} \eta^{-2}\left(1+\xi^{2} \eta^{2}\right)(\xi+\eta)\right)^{2}(\xi+\eta)^{2}} \\
& =\int_{0}^{\infty} \frac{t \eta^{2 \gamma-2}\langle\eta\rangle^{4-4 \gamma}(y-1)^{2} d y}{\left(1+t(y-1)^{2} y^{-2} \eta^{-1}\left(1+\eta^{4} y^{2}\right)(y+1)\right)^{2}(y+1)^{2}} \\
& \leq C t^{-1} \eta^{2 \gamma}\langle\eta\rangle^{4-4 \gamma} \int_{0}^{1 / 2} \frac{y^{4} d y}{\left(1+\eta^{2} y\right)^{4}} \\
& +C t \eta^{2 \gamma-2}\langle\eta\rangle^{4-4 \gamma} \int_{1 / 2}^{3 / 2} \frac{(y-1)^{2} d y}{\left(1+t \eta^{-1}\langle\eta\rangle^{4}(y-1)^{2}\right)^{2}} \\
& +C t^{-1} \eta^{2 \gamma}\langle\eta\rangle^{4-4 \gamma} \int_{3 / 2}^{\infty} \frac{d y}{\left(1+\eta^{2} y\right)^{4} y^{2}} \leq C \eta^{2 \gamma-1}\langle\eta\rangle^{-4 \gamma}, \\
& \int_{0}^{\infty} \frac{t^{1 / 2} \eta^{\gamma-3 / 2}\langle\eta\rangle^{2-2 \gamma} \xi^{-1 / 2} d \xi}{1+t(\xi-\eta)^{2} \xi^{-2} \eta^{-2}\left(1+\xi^{2} \eta^{2}\right)(\xi+\eta)} \\
& =\int_{0}^{\infty} \frac{t^{1 / 2} \eta^{\gamma-1}\langle\eta\rangle^{2-2 \gamma} y^{-1 / 2} d y}{1+t(y-1)^{2} y^{-2} \eta^{-1}\left(1+y^{2} \eta^{4}\right)(y+1)} \\
& \leq C t^{-1 / 2} \eta^{\gamma}\langle\eta\rangle^{2-2 \gamma} \int_{0}^{1 / 2} \frac{y^{3 / 2} d y}{\left(1+\eta^{2} y\right)^{2}} \\
& +C t^{1 / 2} \eta^{\gamma-1}\langle\eta\rangle^{2-2 \gamma} \int_{1 / 2}^{3 / 2} \frac{d y}{1+t \eta^{-1}\langle\eta\rangle^{4}(y-1)^{2}} \\
& +C t^{-1 / 2} \eta^{\gamma}\langle\eta\rangle^{2-2 \gamma} \int_{3 / 2}^{\infty} \frac{y^{-3 / 2} d y}{\left(1+\eta^{2} y\right)^{2}} \leq C \eta^{\gamma-1 / 2}\langle\eta\rangle^{-2 \gamma},
\end{aligned}
$$

we get

$$
\begin{aligned}
& \left\|I_{5}\right\|_{\mathbf{L}^{2}(0, \infty)} \\
& \quad \leq C\left(\left\|\xi \phi_{\xi}\right\|_{\mathbf{L}^{2}}+\left\||\xi|^{1 / 2} \phi\right\|_{\mathbf{L}^{\infty}}\right)\left\|\eta^{\gamma-1 / 2}\langle\eta\rangle^{-2 \gamma}\right\|_{\mathbf{L}^{2}(0, \infty)} \\
& \quad \leq C\left\|\xi \phi_{\xi}\right\|_{\mathbf{L}^{2}(0, \infty)}+C\left\||\xi|^{1 / 2} \phi\right\|_{\mathbf{L}^{\infty}} .
\end{aligned}
$$

In the case of $\eta<0$, the same estimate is obtained easier than the case of the positive line. Lemma 5 is proved.

3.4. Asymptotics for the Nonlinearity. We obtain the asymptotic representation for the nonlinear term. Define the norm

$$
\|\widehat{\varphi}\|_{\mathbf{Z}}=\left\|\langle\xi\rangle^{1 / 2} \hat{\varphi}\right\|_{\mathbf{L}^{\infty}}+\left\|\xi \widehat{\varphi}_{\xi}\right\|_{\mathbf{L}^{2}} .
$$

Lemma 6. The asymptotics

$$
\begin{aligned}
\mathscr{F} \mathcal{U}(-t) \partial_{x} \mathscr{K} u^{3}= & \frac{\sqrt{3} i \xi \widehat{K}}{i t} e^{i t \Omega(\xi)} \mathscr{D}_{3} \frac{1}{\Lambda^{\prime \prime}} \widehat{\varphi}^{3} \\
& +\frac{3 i \xi \widehat{K}}{t \Lambda^{\prime \prime}}|\widehat{\varphi}|^{2} \widehat{\varphi}+O\left(t^{-5 / 4}\|\widehat{\varphi}\|_{\mathrm{Z}}^{3}\right)
\end{aligned}
$$

is true for all $t \geq 1, \xi \geq 0$, where $\widehat{\varphi}(t)=\mathscr{F} \mathcal{U}(-t) u(t)$.
Proof. In view of (37) we find for the new dependent variable $\widehat{\varphi}=\mathscr{F} \mathcal{U}(-t) u(t)$

$$
\begin{aligned}
\mathscr{F} \mathcal{U}(-t) \partial_{x} \mathscr{K} u^{3} \\
=\sqrt{3} i \xi \widehat{K} t^{-1} e^{i t \Omega(\xi)} \mathscr{D}_{3} \mathscr{V}^{*}(3 t) \frac{1}{\Lambda^{\prime \prime}} \psi_{0}^{3} \\
+3 i \xi \widehat{K} t^{-1} \mathscr{V}^{*}(t) \frac{1}{\Lambda^{\prime \prime}} \psi_{0}^{2} \overline{\psi_{0}} \\
+3 i \xi \widehat{K} t^{-1} \mathscr{D}_{-1} \mathscr{V}^{*}(-t) \frac{1}{\Lambda^{\prime \prime}} \psi_{0}{\overline{\psi_{0}}}^{2} \\
+\sqrt{3} i \xi \widehat{K} t^{-1} e^{i t \Omega(\xi)} \mathscr{D}_{-3} \mathscr{V}^{*}(-3 t) \frac{1}{\Lambda^{\prime \prime}} \bar{\psi}_{0}^{3}
\end{aligned}
$$

where $\psi_{j}=\mathscr{V}(i \xi)^{j} \widehat{\varphi}$. By Lemma 3 we have

$$
\begin{aligned}
3 i \xi \widehat{K} t^{-1} \mathscr{V}^{*}(t) \frac{1}{\Lambda^{\prime \prime}} \psi_{0}^{2} \overline{\psi_{0}} \\
=3 i \xi \widehat{K} t^{-1} A^{*}(t) \frac{1}{\Lambda^{\prime \prime}} \psi_{0}^{2} \overline{\psi_{0}} \\
\quad+C t^{-5 / 4}\left\||\eta|^{-9 / 4}\langle\eta\rangle^{4} t \mathscr{A}_{0} \frac{1}{\Lambda^{\prime \prime}} \psi_{0}^{2} \overline{\psi_{0}}\right\|_{\mathbf{L}^{2}} \\
\quad+C t^{-5 / 4}\left\|\frac{1}{\Lambda^{\prime \prime}} \psi_{0}^{2} \overline{\psi_{0}}\right\|_{\mathbf{L}^{\infty}} .
\end{aligned}
$$

By Lemma 2

$$
\begin{aligned}
& \psi_{0} \\
& =A_{0}(t) \hat{\varphi} \\
& +O\left(t^{-1 / 4}|\eta|^{-1 / 4}\langle\eta\rangle^{-1}\left(\left\|\xi^{1 / 2} \widehat{\varphi}\right\|_{L^{\infty}}+\left\|\xi \widehat{\varphi}_{\xi}\right\|_{L^{2}}\right)\right), \\
& \left|\psi_{0}\right| \leq C|\eta|^{-1 / 4}\langle\eta\rangle^{-1 / 4}\|\widehat{\varphi}\|_{\mathbf{Z}} .
\end{aligned}
$$

Therefore

$$
\begin{aligned}
\left\|\frac{1}{\Lambda^{\prime \prime}} \psi_{0}^{2} \overline{\psi_{0}}\right\|_{\mathbf{L}^{\infty}} & \leq C\|\hat{\varphi}\|_{\mathrm{Z}}^{3}\left\|\frac{1}{\Lambda^{\prime \prime}}|\eta|^{-3 / 4}\langle\eta\rangle^{-3 / 4}\right\|_{\mathbf{L}^{\infty}} \\
& \leq C\|\hat{\varphi}\|_{\mathrm{Z}}^{3} .
\end{aligned}
$$

Also by the Leibnitz rule

$$
\begin{aligned}
\mathscr{A}_{0} \frac{1}{\Lambda^{\prime \prime}} \psi_{0}^{2} \overline{\psi_{0}} & =\frac{1}{t \sqrt{\Lambda^{\prime \prime}}} \partial_{\eta}\left(\left(\frac{\psi_{0}}{\sqrt{\Lambda^{\prime \prime}}}\right)^{2} \frac{\overline{\psi_{0}}}{\sqrt{\Lambda^{\prime \prime}}}\right) \\
& =\frac{1}{\Lambda^{\prime \prime}} \psi_{0}^{2} \overline{\mathscr{A}_{0} \psi_{0}}+\frac{2}{\Lambda^{\prime \prime}} \psi_{0} \overline{\psi_{0}} \mathscr{A}_{0} \psi_{0} .
\end{aligned}
$$

Then by Lemma 4 we get

$$
\begin{aligned}
& \left\||\eta|^{-9 / 4}\langle\eta\rangle^{4} t \mathscr{A}_{0} \frac{1}{\Lambda^{\prime \prime}} \psi_{0}^{2} \overline{\psi_{0}}\right\|_{\mathbf{L}^{2}} \\
& \leq C\left\||\eta|^{3 / 4}\left|\psi_{0}\right|^{2} t \mathscr{A}_{0} \psi_{0}\right\|_{\mathbf{L}^{2}} \\
& \leq C\|\hat{\varphi}\|_{\mathbf{Z}}^{2}\left\||\eta|^{1 / 4}\langle\eta\rangle^{-1 / 2} t \mathscr{A}_{0} \psi_{0}\right\|_{\mathbf{L}^{2}} \leq C\|\hat{\varphi}\|_{\mathbf{Z}}^{3} .
\end{aligned}
$$


Hence we obtain

$$
\begin{aligned}
3 i \xi \widehat{K} t^{-1} \mathscr{V}^{*}(t) \frac{1}{\Lambda^{\prime \prime}} \psi_{0}^{2} \overline{\psi_{0}} \\
=3 i \xi \widehat{K} t^{-1} A^{*}(t) \frac{1}{\Lambda^{\prime \prime}}\left(A_{0}(t) \widehat{\varphi}\right)^{2} \overline{A_{0}(t) \widehat{\varphi}} \\
\quad+O\left(t^{-5 / 4}\|\widehat{\varphi}\|_{\mathrm{Z}}^{3}\right) \\
=\frac{3 i \xi \widehat{K}}{t \Lambda^{\prime \prime}}|\widehat{\varphi}|^{2} \widehat{\varphi}+O\left(t^{-5 / 4}\|\widehat{\varphi}\|_{\mathrm{Z}}^{3}\right) .
\end{aligned}
$$

In the same manner

$$
\begin{aligned}
& \sqrt{3} i \xi \widehat{K} t^{-1} e^{i t \Omega(\xi)} \mathscr{D}_{3} \mathscr{V}^{*}(3 t) \frac{1}{\Lambda^{\prime \prime}} \psi_{0}^{3} \\
& =\frac{\sqrt{3} i \xi \widehat{K}}{i t} e^{i t \Omega(\xi)} \mathscr{D}_{3} \frac{1}{\Lambda^{\prime \prime}} \widehat{\varphi}^{3}+O\left(t^{-5 / 4}\|\widehat{\varphi}\|_{\mathrm{Z}}^{3}\right), \\
& 3 i \xi \widehat{K} t^{-1} \mathscr{D}_{-1} \mathscr{V}^{*}(-t) \frac{1}{\Lambda^{\prime \prime}} \psi_{0} \bar{\psi}_{0}^{2}=O\left(t^{-5 / 4}\|\widehat{\varphi}\|_{\mathrm{Z}}^{3}\right), \\
& \sqrt{3} i \xi \widehat{K} t^{-1} e^{i t \Omega(\xi)} \mathscr{D}_{-3} \mathscr{V}^{*}(-3 t) \frac{1}{\Lambda^{\prime \prime}} \bar{\psi}_{0}^{3} \\
& =O\left(t^{-5 / 4}\|\widehat{\varphi}\|_{\mathrm{Z}}^{3}\right) .
\end{aligned}
$$

Hence the result of the lemma follows.

\section{A Priori Estimates}

We define

$$
\begin{aligned}
& \mathbf{X}_{T}=\left\{\mathcal{U}(-t) u \in \mathbf{C}\left([0, T] ; \mathbf{H}^{2}\right) ;\|u\|_{\mathbf{X}_{T}}<\infty\right\}, \\
& \|u\|_{\mathbf{X}_{T}}=\sup _{t \in[0, T]}\left(\left\|\langle\xi\rangle^{1 / 2} \widehat{\varphi}\right\|_{\mathbf{L}^{\infty}}+\langle t\rangle^{-\gamma}\|u(t)\|_{\mathbf{H}^{2}}\right. \\
& \left.\quad+\langle t\rangle^{-\gamma}\left\|\partial_{x} \mathscr{J} u(t)\right\|_{\mathbf{L}^{2}}\right),
\end{aligned}
$$

where $\mathscr{J}=\mathscr{U}(t) x \mathcal{U}(-t), \widehat{\varphi}=\mathscr{F} \mathcal{U}(-t) u(t)$, and $\gamma>0$ is small. We have the local in time existence of solutions.

Theorem 7. Let the initial data $u_{0} \in \mathbf{H}^{2} \cap \mathbf{H}^{1,1}$. Then there exists a time $T_{0}>0$ such that (1) has a unique solution $u$ in $\mathbf{X}_{T_{0}}$.

To get the desired results, we prove a priori estimates of solutions uniformly in time.

Lemma 8. Assume $u_{0} \in \mathbf{H}^{2} \cap \mathbf{H}^{1,1}$ and the norm $\left\|u_{0}\right\|_{\mathbf{H}^{1,1}}+$ $\left\|u_{0}\right\|_{\mathrm{H}^{2}} \leq \varepsilon$. Then the estimate

$$
\|u\|_{\mathbf{X}_{T}}<C \varepsilon
$$

is true for all $T \geq 1$.

Proof. By continuity of the norm $\|u\|_{\mathbf{X}_{T}}$ with respect to $T$, arguing by the contradiction we can find the first time $T \geq$ 1 such that $\|u\|_{\mathbf{X}_{T}}=C \varepsilon$. Consider a priori estimates of $\left\|\partial_{x} \mathscr{J} u(t)\right\|_{\mathrm{L}^{2}}=\left\|\xi \partial_{\xi} \hat{\varphi}\right\|_{\mathbf{L}^{2}}$. To avoid the derivative loss in (1) we apply the operator

$$
\mathscr{P}=t \partial_{t}+\frac{1}{3} x \partial_{x}-\frac{4}{3} a \partial_{a}
$$

and use the commutators $[\mathscr{P}, \mathscr{L}]=-\mathscr{L},\left[\mathscr{P}, \partial_{x} \mathscr{K}\right]=$ $\mathscr{F}^{-1} \widehat{K}_{1}(\xi) \mathscr{F}=\mathscr{K}_{1}$, where $\widehat{K}_{1}(\xi)=(i / 3) \xi \partial_{\xi}(i \xi \widehat{K}(\xi))-$ $(4 / 3) i \xi a \partial_{a} \widehat{K}(\xi)=O(\xi)$. Also we represent $\widehat{K}(\xi)=\lambda+\widehat{K}_{2}(\xi)$ with $\widehat{K}_{2}(\xi)=O\left(\langle\xi\rangle^{-1}\right)$; that is, $\mathscr{K}=\mathscr{K}_{2}+\lambda$. Then we get

$$
\begin{aligned}
\mathscr{L} \mathscr{P} u= & (\mathscr{P}+1) \mathscr{L} u=\mathscr{P} \partial_{x} \mathscr{K} u^{3}+\partial_{x} \mathscr{K} u^{3} \\
= & 3 \partial_{x} \mathscr{K}\left(u^{2} \mathscr{P} u\right)+\partial_{x} \mathscr{K} u^{3}+\left[\mathscr{P}, \partial_{x} \mathscr{K}\right] u^{3} \\
= & 3 \lambda \partial_{x}\left(u^{2} \mathscr{P} u\right)+3 \partial_{x} \mathscr{K}_{2}\left(u^{2} \mathscr{P} u\right)+\partial_{x} \mathscr{K} u^{3} \\
& +\mathscr{K}_{1} u^{3} .
\end{aligned}
$$

Define the high and short frequency projectors $Q_{j} \phi=$ $\mathscr{F}^{-1} \chi_{j} \widehat{\phi}$, where $\chi_{1}(\xi)=1$ for $|\xi| \geq 1$ and $\chi_{1}(\xi)=0$ for $|\xi| \leq 1$, and also $\chi_{2}(\xi)=1-\chi_{1}(\xi)$. Then we get

$$
\begin{aligned}
\mathscr{L} Q_{1} \mathscr{P} u= & 3 \lambda \partial_{x} Q_{1}\left(u^{2} \mathscr{Q}_{1} \mathscr{P} u\right) \\
& +3 \lambda \partial_{x} Q_{1}\left(u^{2} \mathscr{Q}_{2} \mathscr{P} u\right) \\
& +3 \mathscr{Q}_{1} \partial_{x} \mathscr{K}_{2}\left(u^{2} \mathscr{P} u\right)+Q_{1} \partial_{x} \mathscr{K} u^{3} \\
& +Q_{1} \mathscr{K}_{1} u^{3}
\end{aligned}
$$

and the integral equation

$$
\begin{array}{rl}
Q_{2} & \mathscr{P} u=\mathscr{U}(t) \mathscr{Q}_{2} \mathscr{P} u_{0}+\int_{0}^{t} d \tau \mathscr{U}(t-\tau) \\
\cdot\left(3 \lambda \partial_{x} Q_{2}\left(u^{2} Q_{1} \mathscr{P} u\right)+3 \lambda \partial_{x} Q_{2}\left(u^{2} \mathscr{Q}_{2} \mathscr{P} u\right)\right. \\
\left.+3 \mathscr{Q}_{2} \partial_{x} \mathscr{K}_{2}\left(u^{2} \mathscr{P} u\right)+\mathscr{Q}_{2} \partial_{x} \mathscr{K} u^{3}+Q_{2} \mathscr{K}_{1} u^{3}\right) .
\end{array}
$$

Hence applying the energy method to the first equation we find

$$
\begin{aligned}
\frac{d}{d t}\left\|\mathcal{Q}_{1} \mathscr{P} u\right\|_{\mathrm{L}^{2}}^{2} \leq & C\left(\left\|u u_{x}\right\|_{\mathbf{L}^{\infty}}+\|u\|_{\mathbf{L}^{\infty}}^{2}\right) \\
& \cdot\left(\|\mathscr{P} u\|_{\mathbf{L}^{2}}+\|u\|_{\mathbf{L}^{2}}\right)\left\|\mathcal{Q}_{1} \mathscr{P} u\right\|_{\mathbf{L}^{2}}
\end{aligned}
$$

and by the integral equation

$$
\begin{aligned}
& \left\|Q_{2} \mathscr{P} u\right\|_{\mathbf{L}^{2}} \\
& \leq\left\|\mathscr{Q}_{2} \mathscr{P} u_{0}\right\|_{\mathbf{L}^{2}} \\
& \quad+C \int_{0}^{t}\left(\left\|u u_{x}\right\|_{\mathbf{L}^{\infty}}+\|u\|_{\mathbf{L}^{\infty}}^{2}\right)\left(\|\mathscr{P} u\|_{\mathbf{L}^{2}}+\|u\|_{\mathbf{L}^{2}}\right) d \tau .
\end{aligned}
$$

Applying the estimate of Lemma 2 we have

$\left|\mathscr{V} \xi^{j} \phi\right|$

$$
\leq C\langle\eta\rangle^{1 / 2}|\eta|^{-1 / 4}\left(\left\|\langle\xi\rangle^{1 / 2} \hat{\varphi}\right\|_{\mathbf{L}^{\infty}}+t^{-1 / 4}\left\|\xi \phi_{\xi}\right\|_{\mathbf{L}^{2}}\right) .
$$


Hence

$$
\begin{aligned}
\left\|\partial_{x}^{j} u\right\|_{\mathbf{L}^{\infty}} & \leq\left\|2 \operatorname{Re} \mathscr{D}_{t} \mathscr{B} M \mathscr{V}(i \xi)^{j} \hat{\varphi}\right\|_{\mathbf{L}^{\infty}} \\
& \leq C t^{-1 / 2}\left\|\eta^{3 / 2}\langle\eta\rangle^{-2} \mathscr{V} \xi^{j} \hat{\varphi}\right\|_{\mathbf{L}^{\infty}} \\
& \leq C t^{-1 / 2}\left(\left\|\langle\xi\rangle^{1 / 2} \hat{\varphi}\right\|_{\mathbf{L}^{\infty}}+t^{-1 / 4}\left\|\xi \phi_{\xi}\right\|_{\mathbf{L}^{2}}\right) .
\end{aligned}
$$

Therefore

$$
\begin{aligned}
& \frac{d}{d t}\left\|\mathcal{Q}_{1} \mathscr{P} u\right\|_{\mathbf{L}^{2}} \leq C\langle t\rangle^{-1}\left(\left\|\langle\xi\rangle^{1 / 2} \widehat{\varphi}\right\|_{\mathbf{L}^{\infty}}\right. \\
& \left.+t^{-1 / 4}\left\|\xi \phi_{\xi}\right\|_{\mathbf{L}^{2}}\right)\left(\|\mathscr{P} u\|_{\mathbf{L}^{2}}+\|u\|_{\mathbf{L}^{2}}\right), \\
& \left\|\mathcal{Q}_{2} \mathscr{P} u\right\|_{\mathbf{L}^{2}} \leq\left\|\mathcal{Q}_{2} \mathscr{P} u_{0}\right\|_{\mathbf{L}^{2}}+C \int_{0}^{t}\langle t\rangle^{-1} \\
& \cdot\left(\left\|\langle\xi\rangle^{1 / 2} \hat{\varphi}\right\|_{\mathbf{L}^{\infty}}+t^{-1 / 4}\left\|\xi \phi_{\xi}\right\|_{\mathbf{L}^{2}}\right) \\
& \cdot\left(\|\mathscr{P} u\|_{\mathbf{L}^{2}}+\|u\|_{\mathbf{L}^{2}}\right) d \tau .
\end{aligned}
$$

And similarly

$$
\begin{aligned}
& \frac{d}{d t}\|u\|_{\mathbf{H}^{2}} \\
& \quad \leq C\langle t\rangle^{-1}\left(\left\|\langle\xi\rangle^{1 / 2} \hat{\varphi}\right\|_{\mathbf{L}^{\infty}}+t^{-1 / 4}\left\|\xi \phi_{\xi}\right\|_{\mathbf{L}^{2}}\right)\|u\|_{\mathbf{H}^{2}},
\end{aligned}
$$

from which it follows that

$$
\|\mathscr{P} u\|_{\mathbf{L}^{2}}+\|u\|_{\mathbf{H}^{2}} \leq \varepsilon+C \varepsilon^{3}\langle t\rangle^{\gamma} .
$$

By the identity $\mathscr{P}=t \mathscr{L}+(1 / 3) \mathscr{J} \partial_{x}-(4 / 3) a \mathscr{I}$, we obtain

$$
\begin{aligned}
\left\|\xi \partial_{\xi} \hat{\varphi}\right\|_{\mathbf{L}^{2}}= & \left\|\partial_{x} \mathscr{J} u\right\|_{\mathbf{L}^{2}} \\
\leq & C\|\mathscr{P} u\|_{\mathbf{L}^{2}}+t\|\mathscr{L} u\|_{\mathbf{L}^{2}}+C\|\mathscr{I} u\|_{\mathbf{L}^{2}} \\
\leq & C\|\mathscr{P} u\|_{\mathbf{L}^{2}}+C t\left\|u u_{x}\right\|_{\mathbf{L}^{\infty}}\|u\|_{\mathbf{L}^{2}} \\
& +C\|\mathscr{I} u\|_{\mathbf{L}^{2}} \leq C \varepsilon+C \varepsilon^{3} t^{\gamma}+C\|\mathscr{I} u\|_{\mathbf{L}^{2}} .
\end{aligned}
$$

Next we estimate the norm $\|\mathscr{I} u\|_{L^{2}}$. Denote $\widehat{K}_{3}(\xi)=$ $i \xi \partial_{a} \widehat{K}(\xi)=O(1)$. Applying the operator $\mathscr{I}=\partial_{a}-t \partial_{x}^{-1}$ to (1) via the commutator $[\mathscr{I}, \mathscr{L}]=0$, we get

$$
\begin{aligned}
\mathscr{L} \mathscr{I} u= & \mathscr{I} \mathscr{L} u=\mathscr{I} \partial_{x} \mathscr{K} u^{3} \\
= & 3 \lambda \partial_{x}\left(u^{2} \mathscr{I} u\right)+\mathscr{K}_{3} u^{3}+3 i \partial_{x} \mathscr{K}_{2}\left(u^{2} \mathscr{I} u\right) \\
& +3 t \mathscr{K} \partial_{x}\left(u^{2} \partial_{x}^{-1} u\right)-t \mathscr{K} u^{3} \\
= & 3 \lambda \partial_{x}\left(u^{2} \mathscr{I} u\right)+\mathscr{K}_{3} u^{3}+3 \mathscr{K}_{2}\left(u^{2} \mathscr{I} u\right)+N,
\end{aligned}
$$

where $N=2 t \mathscr{K}\left(3 u u_{x} \partial_{x}^{-1} u+u^{3}\right)$. Using the factorization formulas as in the derivation of (37) we find

$$
\begin{aligned}
& \mathscr{F} \mathcal{U}(-t) N=6 t \widehat{K}(\xi) \mathscr{F} \mathcal{U}(-t)\left(u u_{x} \partial_{x}^{-1} u\right) \\
& +2 t \widehat{K}_{1}(\xi) \mathscr{F} \mathcal{U}(-t)\left(u^{3}\right)=6 t \widehat{K}(\xi) \\
& \cdot \mathscr{V}^{*} \bar{M} \mathscr{B}^{-1} \mathscr{D}_{t}^{-1}\left(\mathscr{D}_{t} \mathscr{B}\left(M \psi_{0}+\bar{M} \overline{\psi_{0}}\right)\right) \\
& \cdot\left(\mathscr{D}_{t} \mathscr{B}\left(M \psi_{1}+\bar{M} \overline{\psi_{1}}\right)\right) \\
& \cdot\left(\mathscr{D}_{t} \mathscr{B}\left(M \psi_{-1}+\bar{M} \overline{\psi_{-1}}\right)\right)+2 t \widehat{K}(\xi) \\
& \cdot \mathscr{V}^{*} \bar{M} \mathscr{B}^{-1} \mathscr{D}_{t}^{-1}\left(\mathscr{D} \mathscr{B}_{t}\left(M \psi_{0}+\bar{M} \overline{\psi_{0}}\right)^{3}\right) \\
& =6 \widehat{K}(\xi) \mathscr{V}^{*} \frac{1}{\Lambda^{\prime \prime}} \bar{M}\left(M \psi_{0}+\bar{M} \overline{\psi_{0}}\right)\left(M \psi_{1}+\bar{M} \overline{\psi_{1}}\right) \\
& \cdot\left(M \psi_{-1}+\bar{M} \overline{\psi_{-1}}\right)+2 \widehat{K}(\xi) \mathscr{V}^{*} \frac{1}{\Lambda^{\prime \prime}} \\
& \cdot \bar{M}\left(M \psi_{0}+\bar{M} \overline{\psi_{0}}\right)^{3},
\end{aligned}
$$

where we denote $\psi_{j}=\mathscr{V}(i \xi)^{j} \widehat{\varphi}$. Then we get

$$
\begin{array}{rl}
\mathscr{F} & \mathcal{U}(-t) N=2 \widehat{K}(\xi) \mathscr{V}^{*} M^{2} \frac{1}{\Lambda^{\prime \prime}}\left(3 \psi_{0} \psi_{1} \psi_{-1}+\psi_{0}^{3}\right) \\
+ & 6 \widehat{K}(\xi) \mathscr{V}^{*} \\
& \cdot \frac{1}{\Lambda^{\prime \prime}}\left(\overline{\psi_{0}} \psi_{1} \psi_{-1}+\psi_{0} \overline{\psi_{1}} \psi_{-1}+\psi_{0} \psi_{1} \overline{\psi_{-1}}+\psi_{0}^{2} \overline{\psi_{0}}\right) \\
& +6 \widehat{K}(\xi) \mathscr{V}^{*} \bar{M}^{2} \\
& +\frac{1}{\Lambda^{\prime \prime}}\left(\overline{\psi_{0} \psi_{1}} \psi_{-1}+\overline{\psi_{0}} \psi_{1} \overline{\psi_{-1}}+\psi_{0} \overline{\psi_{1} \psi_{-1}}+\psi_{0}{\overline{\psi_{0}}}^{2}\right) \\
& +2 \widehat{K}(\xi) \mathscr{V}^{*} \bar{M}^{4} \frac{1}{\Lambda^{\prime \prime}}\left(3 \overline{\psi_{0} \psi_{1} \psi_{-1}}+{\overline{\psi_{0}}}^{3}\right) .
\end{array}
$$

Next using identity (36) we find

$$
\begin{array}{rl}
\mathscr{F} & \mathcal{U}(-t) N=2 \sqrt{3} \widehat{K}(\xi) e^{i t \Omega(\xi)} \mathscr{D}_{3} \mathscr{V}^{*}(3 t) \\
& \cdot \frac{1}{\Lambda^{\prime \prime}}\left(3 \psi_{0} \psi_{1} \psi_{-1}+\psi_{0}^{3}\right)+6 \widehat{K}(\xi) \mathscr{V}^{*} \\
& \cdot \frac{1}{\Lambda^{\prime \prime}}\left(\overline{\psi_{0}} \psi_{1} \psi_{-1}+\psi_{0} \overline{\psi_{1}} \psi_{-1}+\psi_{0} \psi_{1} \overline{\psi_{-1}}+\psi_{0}^{2} \overline{\psi_{0}}\right) \\
+ & 6 \widehat{K}(\xi) \mathscr{D}_{-1} \mathscr{V}^{*}(-t) \\
& \cdot \frac{1}{\Lambda^{\prime \prime}}\left(\overline{\psi_{0} \psi_{1}} \psi_{-1}+\overline{\psi_{0}} \psi_{1} \overline{\psi_{-1}}+\psi_{0} \overline{\psi_{1} \psi_{-1}}+\psi_{0}{\overline{\psi_{0}}}^{2}\right) \\
+ & 2 \sqrt{3} \widehat{K}(\xi) e^{i t \Omega(\xi)} \mathscr{D}_{-3} \mathscr{V}^{*}(-3 t) \\
& \cdot \frac{1}{\Lambda^{\prime \prime}}\left(3 \overline{\psi_{0} \psi_{1} \psi_{-1}}+{\overline{\psi_{0}}}^{3}\right)
\end{array}
$$

with $\Omega(\xi)=\Lambda(\xi)-3 \Lambda(\xi / 3)$. Next using the relations $\psi_{j}=$ $\mathscr{V}(i \xi)^{j} \widehat{\varphi}=i \eta \psi_{j-1}+\mathscr{A}_{0} \psi_{j-1}$ and $i \eta \psi_{j}=\psi_{j+1}-\mathscr{A}_{0} \psi_{j}$, we get 
$\psi_{1} \psi_{-1}=\psi_{0}^{2}+R_{1}, \overline{\psi_{1}} \psi_{-1}=-\overline{\psi_{0}} \psi_{0}+R_{2}$, and $\overline{\psi_{1} \psi_{-1}}=\bar{\psi}_{0}^{2}+\overline{R_{1}}$, where $R_{1}=-\psi_{0} \mathscr{A}_{0} \psi_{-1}+\psi_{-1} \mathscr{A}_{0} \psi_{0}$, and $R_{2}=\overline{\psi_{0}} \mathscr{A}_{0} \psi_{-1}+$ $\psi_{-1} \overline{\mathscr{A}_{0} \psi_{0}}$. Therefore we obtain

$$
\begin{aligned}
\mathscr{F} \mathscr{U}(-t) N \\
=8 \sqrt{3} \widehat{K}(\xi) e^{i t \Omega(\xi)} \mathscr{D}_{3} \mathscr{V}^{*}(3 t) \frac{1}{\Lambda^{\prime \prime}} \psi_{0}^{3} \\
\quad+8 \sqrt{3} \widehat{K}(\xi) e^{i t \Omega(\xi)} \mathscr{D}_{-3} \mathscr{V}^{*}(-3 t) \frac{1}{\Lambda^{\prime \prime}} \bar{\psi}_{0}^{3}+R_{3},
\end{aligned}
$$

where

$$
\begin{aligned}
R_{3}= & 6 \sqrt{3} \widehat{K}(\xi) e^{i t \Omega(\xi)} \mathscr{D}_{3} \mathscr{V}^{*}(3 t) \frac{1}{\Lambda^{\prime \prime}} \psi_{0} R_{1}+6 \widehat{K}(\xi) \\
& \cdot \mathscr{V}^{*} \frac{1}{\Lambda^{\prime \prime}}\left(\overline{\psi_{0}} R_{1}+\psi_{0}\left(R_{2}+\overline{R_{2}}\right)\right)+6 \widehat{K}(\xi) \\
& \cdot \mathscr{D}_{-1} \mathscr{V}^{*}(-t) \frac{1}{\Lambda^{\prime \prime}}\left(\psi_{0} \overline{R_{1}}+\overline{\psi_{0}}\left(R_{2}+\overline{R_{2}}\right)\right) \\
+ & 6 \sqrt{3} \widehat{K}(\xi) e^{i t \Omega(\xi)} \mathscr{D}_{-3} \mathscr{V}^{*}(-3 t) \frac{1}{\Lambda^{\prime \prime}} \overline{\psi_{0}} \overline{R_{1}} .
\end{aligned}
$$

By Lemma 2 we have

$$
\begin{aligned}
& \left|\psi_{j}\right| \\
& \quad \leq C|\eta|^{j-1 / 4}\langle\eta\rangle^{1 / 4-j}\left(\left\|\langle\xi\rangle^{1 / 2} \widehat{\varphi}\right\|_{\mathbf{L}^{\infty}}+t^{-1 / 4}\left\|\xi \widehat{\varphi}_{\xi}\right\|_{\mathbf{L}^{2}}\right)
\end{aligned}
$$

for $j=-1,0$, and then by Lemma 4 we obtain

$$
\begin{aligned}
& \left\|\mathcal{U}(t) \mathscr{F}^{-1} R_{3}\right\|_{\mathbf{L}^{2}} \leq\left\|R_{3}\right\|_{\mathbf{L}^{2}} \leq C\left\|\frac{1}{\Lambda^{\prime \prime}}\left|\psi_{0}\right|^{2} \mathscr{A}_{0} \psi_{-1}\right\|_{\mathbf{L}^{2}} \\
& +C\left\|\frac{1}{\Lambda^{\prime \prime}}\left|\psi_{0} \psi_{-1}\right| \mathscr{A}_{0} \psi_{0}\right\|_{\mathbf{L}^{2}} \\
& \leq C\left\|\eta^{4-\gamma}\langle\eta\rangle^{2 \gamma-4}\left|\psi_{0}\right|^{2}\right\|_{\mathbf{L}^{\infty}}\left\|\eta^{\gamma-1}\langle\eta\rangle^{-2 \gamma} \mathscr{A}_{0} \psi_{-1}\right\|_{\mathbf{L}^{2}} \\
& +C\left\|\eta^{3-\gamma}\langle\eta\rangle^{2 \gamma-4}\left|\psi_{0} \psi_{-1}\right|\right\|_{\mathbf{L}^{\infty}}\left\|\eta^{\gamma}\langle\eta\rangle^{-2 \gamma} \mathscr{A}_{0} \psi_{0}\right\|_{\mathbf{L}^{2}} \\
& \leq C t^{-1}\left(\left\|\langle\xi\rangle^{1 / 2} \hat{\varphi}\right\|_{\mathbf{L}^{\infty}}+t^{-1 / 4}\left\|\xi \hat{\varphi}_{\xi}\right\|_{\mathbf{L}^{2}}\right)^{2} \\
& \cdot\left(\left\|\xi \widehat{\varphi}_{\xi}\right\|_{\mathbf{L}^{2}}+\left\|\xi^{1 / 2} \hat{\varphi}\right\|_{\mathbf{L}^{\infty}}\right) .
\end{aligned}
$$

Then we represent

$$
\begin{aligned}
\mathscr{F} \mathscr{U}(-t) N \\
=8 \sqrt{3} \widehat{K}(\xi) e^{i t \Omega(\xi)} \mathscr{D}_{3} \mathscr{V}^{*}(3 t) \frac{1}{\Lambda^{\prime \prime}} \psi_{0}^{3} \\
\quad+8 \sqrt{3} \widehat{K}(\xi) e^{i t \Omega(\xi)} \mathscr{D}_{-3} \mathscr{V}^{*}(-3 t) \frac{1}{\Lambda^{\prime \prime}} \bar{\psi}_{0}^{3}+R_{3} \\
=\partial_{t} \Psi+R_{3}+R_{4},
\end{aligned}
$$

where

$$
\begin{aligned}
\Psi & =8 \sqrt{3} \frac{\widehat{K}(\xi)}{i \Omega(\xi)} e^{i t \Omega(\xi)}\left(\mathscr{D}_{3} \mathscr{V}^{*}(3 t) \frac{1}{\Lambda^{\prime \prime}} \psi_{0}^{3}\right. \\
& \left.+\mathscr{D}_{-3} \mathscr{V}^{*}(-3 t) \frac{1}{\Lambda^{\prime \prime}} \bar{\psi}_{0}^{3}\right), \\
R_{4} & =8 \sqrt{3} \frac{\widehat{K}(\xi)}{i \Omega(\xi)} e^{i t \Omega(\xi)} \partial_{t}\left(\mathscr{D}_{3} \mathscr{V}^{*}(3 t) \frac{1}{\Lambda^{\prime \prime}} \psi_{0}^{3}\right. \\
& \left.+\mathscr{D}_{-3} \mathscr{V}^{*}(-3 t) \frac{1}{\Lambda^{\prime \prime}} \bar{\psi}_{0}^{3}\right) .
\end{aligned}
$$

We need to estimate the derivative $\mathscr{V}_{t}^{*}$. We have

$$
\begin{aligned}
\mathscr{V}_{t}^{*} \phi & \\
= & \frac{1}{2 t} \mathscr{V}^{*} \phi \\
& +\frac{|t|^{1 / 2}}{\sqrt{2 \pi}} \int_{0}^{\infty} e^{i t S(\eta, \xi)} i S(\eta, \xi) \phi(\eta) \sqrt{\Lambda^{\prime \prime}(\eta)} d \eta .
\end{aligned}
$$

Since $S(\eta, \xi)=\Lambda(\xi)-\Lambda(\eta)-\Lambda^{\prime}(\eta)(\xi-\eta)=(1 / 3) \xi^{-1} \eta^{-2}(3 a+$ $\left.2 b \eta^{3} \xi+b \eta^{2} \xi^{2}\right)(\xi-\eta)^{2}$ and also $i \xi \mathscr{V}^{*}(t) \phi-\mathscr{V}^{*}(t) i \eta \phi=$ $\mathscr{V}^{*}(t) \mathscr{A}_{0}(t) \phi$ we find for the second summand

$$
\begin{gathered}
\frac{|t|^{1 / 2}}{\sqrt{2 \pi}} \int_{0}^{\infty} e^{i t S(\eta, \xi)} i S(\eta, \xi) \phi(\eta) \sqrt{\Lambda^{\prime \prime}(\eta)} d \eta=\frac{i}{3} \frac{|t|^{1 / 2}}{\sqrt{2 \pi}} \\
\cdot \int_{0}^{\infty} e^{i t S(\eta, \xi)}\left(b \eta \xi+3 \frac{a}{\eta^{2}}-2 b \eta^{2}+b \xi^{2}-3 \frac{a}{\eta \xi}\right) \\
\cdot(\xi-\eta) \phi(\eta) \sqrt{\Lambda^{\prime \prime}(\eta)} d \eta=\frac{i b}{3} \xi \mathscr{V}^{*}(t) \eta \mathscr{A}_{0}(t) \phi \\
\quad+i a \mathscr{V}^{*}(t) \eta^{-2} \mathscr{A}_{0}(t) \phi-\frac{2 i}{3} b \mathscr{V}^{*}(t) \eta^{2} \mathscr{A}_{0}(t) \phi \\
+\frac{i}{3} b \xi^{2} \mathscr{V}^{*}(t) \mathscr{A}_{0}(t) \phi-i a \xi^{-1} \mathscr{V}^{*}(t) \eta^{-1} \mathscr{A}_{0}(t) \phi
\end{gathered}
$$

Since $\partial_{t} \psi_{0}=\mathscr{V}_{t} \widehat{\varphi}+\mathscr{V} \widehat{\varphi_{t}}$, we obtain

$$
\begin{aligned}
& \left\|\mathcal{U}(t) \mathscr{F}^{-1} R_{4}\right\|_{\mathbf{L}^{2}} \leq\left\|R_{4}\right\|_{\mathbf{L}^{2}} \\
& \leq\left\|\xi\langle\xi\rangle^{-4} \mathscr{V}_{t}^{*}(3 t) \frac{1}{\Lambda^{\prime \prime}} \psi_{0}^{3}\right\|_{\mathbf{L}^{2}} \\
& +\left\|\mathscr{V}^{*}(3 t) \frac{1}{\Lambda^{\prime \prime}} \psi_{0}^{2} \partial_{t} \psi_{0}\right\|_{\mathbf{L}^{2}} \\
& \leq C t^{-1}\left\|\frac{1}{\Lambda^{\prime \prime}} \psi_{0}^{3}\right\|_{\mathbf{L}^{2}} \\
& +C\left\|\left(\eta^{2}+\eta^{-2}\right) \mathscr{A}_{0}(3 t) \frac{1}{\Lambda^{\prime \prime}} \psi_{0}^{3}\right\|_{\mathbf{L}^{2}} \\
& +C\left\|\frac{1}{\Lambda^{\prime \prime}}\left|\psi_{0}\right|^{2} \partial_{t} \psi_{0}\right\|_{\mathbf{L}^{2}} \cdot
\end{aligned}
$$


By Lemmas 4 and 5

$$
\begin{aligned}
& \left\|\frac{1}{\Lambda^{\prime \prime}} \psi_{0}^{3}\right\|_{\mathbf{L}^{2}} \leq C\|\widehat{\varphi}\|_{\mathbf{Z}}^{3}\left\|\frac{1}{\Lambda^{\prime \prime}}|\eta|^{-3 / 4}\langle\eta\rangle^{-3 / 4}\right\|_{\mathbf{L}^{2}} \\
& \leq C\|\hat{\varphi}\|_{\mathbf{Z}}^{3}, \\
& \left\|\left(\eta^{2}+\eta^{-2}\right) \mathscr{A}_{0}(3 t) \frac{1}{\Lambda^{\prime \prime}} \psi_{0}^{3}\right\|_{\mathbf{L}^{2}} \leq C\left\|\eta\left|\psi_{0}\right|^{2} \mathscr{A}_{0} \psi_{0}\right\|_{\mathbf{L}^{2}} \\
& \leq C t^{-1}\|\hat{\varphi}\|_{\mathbf{Z}}^{2}\left\||\eta|^{1 / 4}\langle\eta\rangle^{-1 / 2} t \mathscr{A}_{0} \psi_{0}\right\|_{\mathbf{L}^{2}} \\
& \leq C t^{-1}\|\hat{\varphi}\|_{\mathbf{Z}}^{3}, \\
& \left\|\frac{1}{\Lambda^{\prime \prime}}\left|\psi_{0}\right|^{2} \partial_{t} \psi_{0}\right\|_{\mathbf{L}^{2}} \leq C t^{-1}\left\|\frac{1}{\Lambda^{\prime \prime}}\left|\psi_{0}\right|^{2} \eta^{-\gamma}\langle\eta\rangle^{2 \gamma+2}\right\|_{\mathbf{L}^{\infty}} \\
& \cdot\left\|\eta^{\gamma}\langle\eta\rangle^{-2 \gamma-2} t \partial_{t} \psi_{0}\right\|_{\mathbf{L}^{2}} \leq C t^{-1}\|\hat{\varphi}\|_{\mathbf{Z}}^{3} \cdot
\end{aligned}
$$

Therefore

$$
\left\|\mathcal{U}(t) \mathscr{F}^{-1} R_{4}\right\|_{\mathrm{L}^{2}} \leq C t^{-1}\|\hat{\varphi}\|_{\mathrm{Z}}^{3}
$$

Thus we get

$$
\begin{aligned}
\mathscr{L} \mathscr{I} u= & 3 \lambda \partial_{x}\left(u^{2} \mathscr{I} u\right)+\mathscr{K}_{3} u^{3}+3 \mathscr{K}_{2}\left(u^{2} \mathscr{I} u\right) \\
& +\mathscr{U}(t) \mathscr{F}^{-1} \partial_{t} \Psi+\mathscr{U}(t) \mathscr{F}^{-1}\left(R_{3}+R_{4}\right) \\
= & 3 \lambda \partial_{x}\left(u^{2} \mathscr{I} u\right)+\mathscr{K}_{3} u^{3}+3 \mathscr{K}_{2}\left(u^{2} \mathscr{I} u\right) \\
& +\mathscr{L} \mathcal{U}(t) \mathscr{F}^{-1} \Psi+\mathscr{U}(t) \mathscr{F}^{-1}\left(R_{3}+R_{4}\right) .
\end{aligned}
$$

Hence

$$
\begin{aligned}
\mathscr{L}\left(\mathscr{I} u-\mathscr{U}(t) \mathscr{F}^{-1} \Psi\right) \\
=3 \lambda \partial_{x}\left(u^{2}\left(\mathscr{I} u-\mathscr{U}(t) \mathscr{F}^{-1} \Psi\right)\right) \\
\quad+3 \lambda \partial_{x}\left(u^{2}\left(\mathscr{U}(t) \mathscr{F}^{-1} \Psi\right)\right)+\mathscr{K}_{3} u^{3} \\
\quad+3 \mathscr{K}_{2}\left(u^{2} \mathscr{I} u\right)+\mathscr{U}(t) \mathscr{F}^{-1}\left(R_{3}+R_{4}\right) .
\end{aligned}
$$

Then as the above using the projectors $\mathbb{Q}_{1}$ and $\mathbb{Q}_{2}$ we find

$$
\left\|\mathscr{I} u-\mathscr{U}(t) \mathscr{F}^{-1} \Psi\right\|_{\mathbf{L}^{2}} \leq C \varepsilon+C \varepsilon^{3} t^{\gamma-1}
$$

We have

$$
\begin{aligned}
\left\|\mathcal{U}(t) \mathscr{F}^{-1} \Psi\right\|_{\mathbf{L}^{2}} & \leq\|\Psi\|_{\mathbf{L}^{2}} \leq C\left\|\eta^{3}\langle\eta\rangle^{-7} \psi_{0}^{3}\right\|_{\mathbf{L}^{2}} \\
& \leq C \varepsilon^{3} t^{\gamma-1},
\end{aligned}
$$

and then we get

$$
\|\mathscr{I} u\|_{\mathbf{L}^{2}} \leq C \varepsilon+C \varepsilon^{3} t^{\gamma-1}
$$

Therefore

$$
\left\|\xi \partial_{\xi} \widehat{\varphi}\right\|_{\mathbf{L}^{2}} \leq 5 \varepsilon+C \varepsilon^{3} t^{\gamma}
$$

Next we estimate $\left\|\langle\xi\rangle^{1 / 2} \widehat{\varphi}\right\|_{L^{\infty}}$. In the domain $|\xi| \geq\langle t\rangle^{\nu}$ we get by the Sobolev imbedding theorem

$$
\begin{aligned}
& \left\|\langle\xi\rangle^{1 / 2} \widehat{\varphi}(t, \xi)\right\|_{\mathbf{L}^{\infty}\left(|\xi| \geq\langle t\rangle^{\nu}\right)} \\
& \quad \leq C\langle t\rangle^{-v}\left\|\langle\xi\rangle^{3 / 2} \widehat{\varphi}(t, \xi)\right\|_{\mathbf{L}^{\infty}\left(|\xi| \geq\langle t\rangle^{\nu}\right)} \\
& \quad \leq C\langle t\rangle^{-v}\left(\left\|\xi \partial_{\xi} \widehat{\varphi}\right\|_{\mathbf{L}^{2}}+\left\|\langle\xi\rangle^{2} \widehat{\varphi}\right\|_{\mathbf{L}^{2}}\right) \leq C \varepsilon\langle t\rangle^{-\nu+\gamma},
\end{aligned}
$$

if $\nu>\gamma$, so we need to estimate the function $\langle\xi\rangle^{(1 / 2)} \widehat{\varphi}(t, \xi)$ in the domain $|\xi| \leq\langle t\rangle^{\nu}$. Next by (37) for $\widehat{\varphi}=\mathscr{F} \mathcal{U}(-t) u(t)$, using Lemma 6, we get

$$
\begin{aligned}
\partial_{t} \widehat{\varphi}(t, \xi)= & \mathscr{F} \mathcal{U}(-t) \partial_{x} \mathscr{K} u^{3} \\
= & \frac{\sqrt{3} i \xi \widehat{K}}{i t} e^{i t \Omega(\xi)} \mathscr{D}_{3} \frac{1}{\Lambda^{\prime \prime}} \widehat{\varphi}^{3}+\frac{3 i \xi \widehat{K}}{t \Lambda^{\prime \prime}}|\widehat{\varphi}|^{2} \widehat{\varphi} \\
& +O\left(t^{-5 / 4}\|\widehat{\varphi}\|_{\mathrm{Z}}^{3}\right)
\end{aligned}
$$

for all $t \geq 1,|\xi| \leq\langle t\rangle^{\nu}$. Multiplying this formula by $\langle\xi\rangle^{1 / 2}$ we get

$$
\begin{aligned}
\partial_{t}\langle\xi\rangle^{1 / 2} \widehat{\varphi}(t, \xi)= & \frac{\sqrt{3} i \xi \widehat{K}}{i t}\langle\xi\rangle^{1 / 2} e^{i t \Omega(\xi)} \mathscr{D}_{3} \frac{1}{\Lambda^{\prime \prime}} \widehat{\varphi}^{3} \\
& +\frac{3 i \xi \widehat{K}}{t \Lambda^{\prime \prime}}\langle\xi\rangle^{1 / 2}|\widehat{\varphi}|^{2} \widehat{\varphi} \\
& +O\left(t^{\nu-5 / 4}\|\hat{\varphi}\|_{\mathbf{Z}}^{3}\right)
\end{aligned}
$$

in the domain $|\xi| \leq\langle t\rangle^{\nu}$. Define the cut-off function $\chi \epsilon$ $\mathbf{C}^{1}(\mathbf{R})$, such that $\chi(x)=1$ for $|x|<1$ and $\chi(x)=0$ for $|x|>2$, and define $\widehat{\varphi}_{1}(t, \xi)=\chi\left(\xi\langle t\rangle^{-v}\right)\langle\xi\rangle^{1 / 2} \widehat{\varphi}(t, \xi)$. Thus we get

$$
\begin{aligned}
\partial_{t} \widehat{\varphi}_{1}= & \frac{\sqrt{3} i \xi \widehat{K}}{i t} \chi\left(\xi\langle t\rangle^{-v}\right)\langle\xi\rangle^{1 / 2} e^{i t \Omega(\xi)} \mathscr{D}_{3} \frac{1}{\Lambda^{\prime \prime}} \widehat{\varphi}^{3} \\
& +\frac{3 i \xi \widehat{K}}{t \Lambda^{\prime \prime}}|\widehat{\varphi}|^{2} \widehat{\varphi}_{1} \\
& +\langle\xi\rangle^{1 / 2} \xi\langle t\rangle^{-1-\nu} \chi^{\prime}\left(\xi\langle t\rangle^{-v}\right) \widehat{\varphi}(t, \xi) \\
& +O\left(t^{\nu-5 / 4}\|\widehat{\varphi}\|_{\mathrm{Z}}^{3}\right)
\end{aligned}
$$

for all $t \geq 1$. The third term is estimated by $C \varepsilon\langle t\rangle^{-1-\nu+\gamma}$. To exclude the resonant term we make a change $\widehat{\varphi}_{1}(t, \xi)=$ $y(t, \xi) \Theta(t, \xi)$, where

$$
\Theta(t, \xi)=\exp \left(\frac{3 i \xi \widehat{K}}{\Lambda^{\prime \prime}} \int_{1}^{t}|\widehat{\varphi}(\tau, \xi)|^{2} \frac{d \tau}{\tau}\right) .
$$

Then we get

$$
\begin{aligned}
y_{t}(t, \xi) & \\
= & \frac{\sqrt{3} i \xi \widehat{K}}{i t} \chi\left(\xi\langle t\rangle^{-\nu}\right)\langle\xi\rangle^{1 / 2} e^{i t \Omega(\xi)} \overline{\Theta(t, \xi)} \mathscr{D}_{3} \frac{1}{\Lambda^{\prime \prime}} \widehat{\varphi}^{3} \\
& +O\left(\varepsilon^{3} t^{-1-\delta}\right)
\end{aligned}
$$


with $\delta>0$. Integrating by parts we obtain

$$
\begin{aligned}
& y(t, \xi)-y(1, \xi)=\int_{1}^{t} \sqrt{3} i \xi \widehat{K} \chi\left(\xi\langle\tau\rangle^{-v}\right)\langle\xi\rangle^{1 / 2} \\
& \cdot e^{i \tau \Omega(\xi)} \overline{\Theta(\tau, \xi)} \mathscr{D}_{3} \frac{1}{\Lambda^{\prime \prime}} \widehat{\varphi}^{3} \frac{d \tau}{i \tau}+O\left(\varepsilon^{3}\right)=\sqrt{3} \\
& \cdot \frac{i \xi \widehat{K}}{i \Omega(\xi)} e^{i \tau \Omega(\xi)} \chi\left(\xi\langle\tau\rangle^{-v}\right)\langle\xi\rangle^{1 / 2} \overline{\Theta(\tau, \xi)} \mathscr{D}_{3} \frac{1}{\tau \Lambda^{\prime \prime}} \\
& \left.\cdot \widehat{\varphi}^{3}\right|_{\tau=1} ^{\tau=t}+\int_{1}^{t} \sqrt{3} \frac{i \xi \widehat{K}}{i \Omega(\xi)} e^{i \tau \Omega(\xi)} \partial_{\tau}\left(\chi\left(\xi\langle\tau\rangle^{-v}\right)\right. \\
& \left.\cdot\langle\xi\rangle^{1 / 2} \overline{\Theta(\tau, \xi)} \mathscr{D}_{3} \frac{1}{\tau \Lambda^{\prime \prime}} \widehat{\varphi}^{3}\right) d \tau+O\left(\varepsilon^{3}\right) \\
& =O\left(\varepsilon^{3}\right) .
\end{aligned}
$$

Thus we get the estimate $\left|\widehat{\varphi}_{1}(\xi)\right|=|y(t, \xi)| \leq\left|\widehat{\varphi}_{1}(1, \xi)\right|+O\left(\varepsilon^{3}\right)$ in the domain $|\xi| \leq\langle t\rangle^{\nu}$. Therefore we find the desired estimate $\left\|\langle\xi\rangle^{1 / 2} \hat{\varphi}\right\|_{\mathbf{L}^{\infty}} \leq C \boldsymbol{\varepsilon}$. This is the desired contradiction. Lemma 8 is proved.

\section{Proof of Theorem 1}

The global existence of solution $u \in \mathbf{C}\left([0, T] ; \mathbf{H}^{2}\right)$ to Cauchy problem (1) satisfying a priori estimate

$$
\begin{aligned}
& \left\|\langle\xi\rangle^{1 / 2} \widehat{\varphi}\right\|_{\mathbf{L}^{\infty}}+\langle t\rangle^{-\gamma}\|u(t)\|_{\mathbf{H}^{2}}+\langle t\rangle^{-\gamma}\left\|\partial_{x} \mathscr{J} u(t)\right\|_{\mathbf{L}^{2}} \\
& \quad \leq C \varepsilon
\end{aligned}
$$

follows by a standard continuation argument from Lemma 8 and local existence Theorem 7. We need only to prove asymptotic formula (20).

We need to compute the asymptotics of the function $\widehat{\varphi}(t, \xi)$. As in the proof of Lemma 8 we get

$$
\begin{aligned}
& y(t, \xi)-y(s, \xi)=\int_{s}^{t} \sqrt{3} i \xi \widehat{K} \chi\left(\xi\langle\tau\rangle^{-v}\right)\langle\xi\rangle^{1 / 2} \\
& \cdot e^{i \tau \Omega(\xi)} \overline{\Theta(\tau, \xi)} \mathscr{D}_{3} \frac{1}{\Lambda^{\prime \prime}} \widehat{\varphi}^{3} \frac{d \tau}{i \tau}+O\left(\varepsilon^{3} s^{-\delta}\right)=\sqrt{3} \\
& \cdot \frac{i \xi \widehat{K}}{i \Omega(\xi)} e^{i \tau \Omega(\xi)} \chi\left(\xi\langle\tau\rangle^{-v}\right)\langle\xi\rangle^{1 / 2} \overline{\Theta(\tau, \xi)} \mathscr{D}_{3} \frac{1}{\tau \Lambda^{\prime \prime}} \\
& \left.\cdot \widehat{\varphi}^{3}\right|_{\tau=s} ^{\tau=t}+\int_{s}^{t} \sqrt{3} \frac{i \xi \widehat{K}}{i \Omega(\xi)} e^{i \tau \Omega(\xi)} \partial_{\tau}\left(\chi\left(\xi\langle\tau\rangle^{-\nu}\right)\right. \\
& \left.\cdot\langle\xi\rangle^{1 / 2} \overline{\Theta(\tau, \xi)} \mathscr{D}_{3} \frac{1}{\tau \Lambda^{\prime \prime}} \widehat{\varphi}^{3}\right) d \tau+O\left(\varepsilon^{3} s^{-\delta}\right) \\
& =O\left(\varepsilon^{3} s^{-\delta}\right)
\end{aligned}
$$

for any $t>s>0$. Therefore there exists a unique final state $y_{+} \in \mathbf{L}^{\infty}$ such that

$$
\left\|y(t)-y_{+}\right\|_{\mathbf{L}^{\infty}} \leq C \varepsilon^{3} t^{-\delta}
$$

for all $t>0$. We write

$$
\begin{aligned}
\frac{3 \widehat{K}}{\Lambda^{\prime \prime}} \int_{1}^{t}|\widehat{\varphi}(\tau, \xi)|^{2} \frac{d \tau}{\tau} & =\frac{3 i \xi \widehat{K}}{\langle\xi\rangle \Lambda^{\prime \prime}} \int_{1}^{t}|y(\tau, \xi)|^{2} \frac{d \tau}{\tau} \\
& =\frac{3 i \xi \widehat{K}}{\langle\xi\rangle \Lambda^{\prime \prime}}\left|y_{+}\right|^{2} \log t+\Phi_{2}(t) .
\end{aligned}
$$

We study the asymptotics in time of the remainder term $\Phi_{2}(t)$. We have

$$
\begin{aligned}
\Phi_{2}(t)-\Phi_{2}(s)= & \frac{3 i \xi \widehat{K}}{\langle\xi\rangle \Lambda^{\prime \prime}} \int_{s}^{t}\left(|y(\tau)|^{2}-|y(t)|^{2}\right) \frac{d \tau}{\tau} \\
& +\frac{3 i \xi \widehat{K}}{\langle\xi\rangle \Lambda^{\prime \prime}}\left(|y(t)|^{2}-\left|y_{+}\right|^{2}\right) \log \frac{t}{s}
\end{aligned}
$$

By (185) we obtain $\left\|\Phi_{2}(t)-\Phi_{2}(s)\right\|_{L^{\infty}} \leq C \varepsilon^{3} s^{-\delta}$ for any $t>s>$ 0 . Hence there exists a unique real-valued function $\Phi_{+} \in \mathbf{L}^{\infty}$ such that

$$
\left\|\Phi_{2}(t)-\Phi_{+}\right\|_{\mathbf{L}^{\infty}} \leq C \varepsilon^{3} t^{-\delta}
$$

for all $t>0$. Representation (186) and estimate (188) yield

$$
\begin{aligned}
& \left\|\Theta(\tau, \xi)-\exp \left(\frac{3 i \xi \widehat{K}}{\langle\xi\rangle \Lambda^{\prime \prime}}\left|y_{+}\right|^{2} \log t+\Phi_{+}\right)\right\|_{\mathbf{L}^{\infty}} \\
& \quad \leq C t^{-\delta}
\end{aligned}
$$

for all $t>0$. Thus we get the large time asymptotics

$$
\begin{aligned}
& \left\|\chi\left(\xi\langle t\rangle^{-v}\right)\langle\xi\rangle^{1 / 2} \widehat{\varphi}(t, \xi)-y_{+} \Theta(\tau, \xi)\right\|_{\mathbf{L}^{\infty}} \\
& \quad=\left\|y(t)-y_{+}\right\|_{\mathbf{L}^{\infty}} \leq C t^{-\delta}, \\
& \left\|y_{+} \Theta(\tau, \xi)-y_{+} \exp \left(\frac{3 i \xi \widehat{K}}{\langle\xi\rangle \Lambda^{\prime \prime}}\left|y_{+}\right|^{2} \log t+\Phi_{+}\right)\right\|_{\mathbf{L}^{\infty}} \\
& \quad \leq C t^{-\delta} .
\end{aligned}
$$

Therefore we obtain the estimate

$$
\begin{aligned}
& \| \chi\left(\xi\langle t\rangle^{-v}\right) \hat{\varphi}(t, \xi) \\
& -W_{+} \exp \left(\frac{3 i \xi \widehat{K}}{\langle\xi\rangle \Lambda^{\prime \prime}}\left|W_{+}\right|^{2} \log t\right) \|_{L^{\infty}} \leq C t^{-\delta}
\end{aligned}
$$


with $W_{+}=y_{+} e^{\Phi_{+}}$. Using the factorization of $\mathscr{U}(t)$ we have

$$
\begin{aligned}
& \| u(t) \\
& -2 \operatorname{Re} \mathscr{D}_{t} \mathscr{B}^{-1} M W_{+} \exp \left(\frac{3 i \xi \widehat{K}}{\langle\xi\rangle \Lambda^{\prime \prime}}\left|W_{+}\right|^{2} \log t\right) \|_{\mathbf{L}^{\infty}} \\
& \leq C \| \mathscr{D}_{t} \mathscr{B}^{-1} M\left(\chi\left(\xi\langle t\rangle^{-v}\right) \widehat{\varphi}(t, \xi)\right. \\
& \left.-W_{+} \exp \left(\frac{3 i \xi \widehat{K}}{\langle\xi\rangle \Lambda^{\prime \prime}}\left|W_{+}\right|^{2} \log t\right)\right) \|_{\mathbf{L}^{\infty}}+C t^{-1 / 2-\delta} \\
& \leq C t^{-1 / 2-\delta} .
\end{aligned}
$$

This completes the proof of asymptotics (20). Theorem 1 is proved.

\section{Competing Interests}

The authors declare that they have no competing interests.

\section{Acknowledgments}

The work of Nakao Hayashi is partially supported by JSPS KAKENHI Grant nos. JP25220702 and JP15H03630. The work of Pavel I. Naumkin is partially supported by CONACYT and PAPIIT Project IN100616.

\section{References}

[1] N. Hayashi and P. I. Naumkin, "Large time asymptotics for the reduced Ostrovsky equation," Communications in Mathematical Physics, vol. 335, no. 2, pp. 713-738, 2015.

[2] L. A. Ostrovsky and Y. A. Stepanyants, "Nonlinear surface and internal waves in rotating fluids," in Nonlinear Waves 3, Research Reports in Physics, pp. 106-128, Springer, Berlin, Germany, 1990.

[3] F. Linares and A. Milanes, "Local and global well-posedness for the Ostrovsky equation," Journal of Differential Equations, vol. 222, no. 2, pp. 325-340, 2006.

[4] K. Tsugawa, "Well-posedness and weak rotation limit for the Ostrovsky equation," Journal of Differential Equations, vol. 247, no. 12, pp. 3163-3180, 2009.

[5] V. Varlamov and Y. Liu, "Cauchy problem for the Ostrovsky equation," Discrete and Continuous Dynamical Systems Series A, vol. 10, no. 3, pp. 731-753, 2004.

[6] G. Gui and Y. Liu, "On the Cauchy problem for the Ostrovsky equation with positive dispersion," Communications in Partial Differential Equations, vol. 32, no. 10-12, pp. 1895-1916, 2007.

[7] Z. Huo and Y. Jia, "Low-regularity solutions for the Ostrovsky equation," Proceedings of the Edinburgh Mathematical Society, vol. 49, no. 1, pp. 87-100, 2006.

[8] P. Isaza and J. Mejía, "Cauchy problem for the Ostrovsky equation in spaces of low regularity," Journal of Differential Equations, vol. 230, no. 2, pp. 661-681, 2006.

[9] J. Bourgain, "Fourier transform restriction phenomena for certain lattice subsets and applications to nonlinear evolution equations. II. The KdV-equation," Geometric and Functional Analysis, vol. 3, no. 3, pp. 209-262, 1993.

[10] J. Colliander, M. Keel, G. Staffilani, H. Takaoka, and T. Tao, "Sharp global well-posedness for KdV and modified KdV on $\mathbb{R}$ and T," Journal of the American Mathematical Society, vol. 16, no. 3, pp. 705-749, 2003.

[11] T. Schäfer and C. E. Wayne, "Propagation of ultra-short optical pulses in cubic nonlinear media," Physica D. Nonlinear Phenomena, vol. 196, no. 1-2, pp. 90-105, 2004.

[12] A. Stefanov, Y. Shen, and P. G. Kevrekidis, "Well-posedness and small data scattering for the generalized Ostrovsky equation," Journal of Differential Equations, vol. 249, no. 10, pp. 2600-2617, 2010.

[13] Y. Liu, D. Pelinovsky, and A. Sakovich, "Wave breaking in the short-pulse equation," Dynamics of Partial Differential Equations, vol. 6, no. 4, pp. 291-310, 2009.

[14] Y. Liu, D. Pelinovsky, and A. Sakovich, "Wave breaking in the Ostrovsky-Hunter equation," SIAM Journal on Mathematical Analysis, vol. 42, no. 5, pp. 1967-1985, 2010.

[15] D. Pelinovsky and A. Sakovich, "Global well-posedness of the short-pulse and sine-Gordon equations in energy space," Communications in Partial Differential Equations, vol. 35, no. 4, pp. 613-629, 2010.

[16] J. P. Boyd, “Ostrovsky and Hunter's generic wave equation for weakly dispersive waves: matched asymptotic and pseudospectral study of the paraboloidal travelling waves (corner and nearcorner waves)," European Journal of Applied Mathematics, vol. 16, no. 1, pp. 65-81, 2005.

[17] J. Hunter, "Numerical solutions of some nonlinear dispersive wave equations," Lectures in Applied Mathematics, vol. 26, pp. 301-316, 1990.

[18] R. Grimshaw and D. Pelinovsky, "Global existence of smallnorm solutions in the reduced Ostrovsky equation," Discrete and Continuous Dynamical Systems Series A, vol. 34, no. 2, pp. 557-566, 2014.

[19] N. Hayashi, P. I. Naumkin, and T. Niizato, "Asymptotics of solutions to the generalized Ostrovsky equation," Journal of Differential Equations, vol. 255, no. 8, pp. 2505-2520, 2013.

[20] N. Hayashi, P. I. Naumkin, and T. Niizato, "Nonexistence of the usual scattering states for the generalized Ostrovsky-Hunter equation," Journal of Mathematical Physics, vol. 55, no. 5, Article ID 053502, 2014.

[21] M. V. Fedoryuk, Asymptotics: Integrals and Series, Mathematical Reference Library, Nauka, Moscow, Russia, 1987.

[22] M. V. Fedoryuk, "Asymptotic methods in analysis," in Analysis I, vol. 13 of Encyclopaedia of Mathematical Sciences, pp. 83-191, Springer, New York, NY, USA, 1989.

[23] E. M. Stein and R. Shakarchi, Functional Analysis. Introduction to Further Topics in Analysis, vol. 4 of Princeton Lectures in Analysis, Princeton University Press, Princeton, NJ, USA, 2011. 


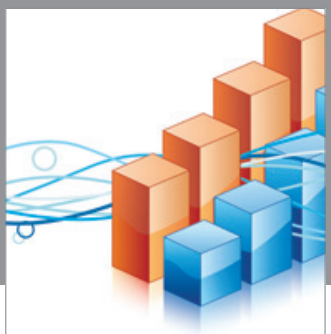

Advances in

Operations Research

vatem alat4

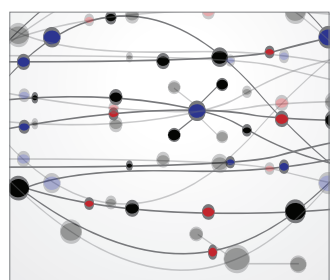

\section{The Scientific} World Journal
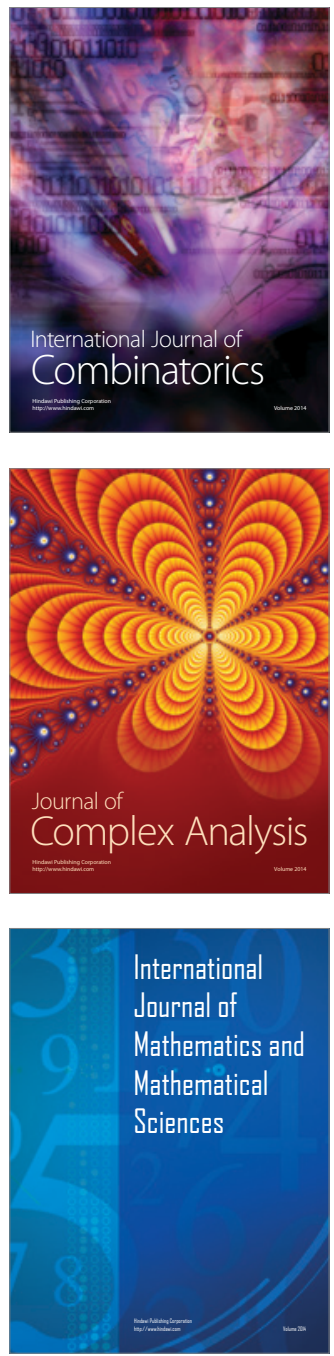
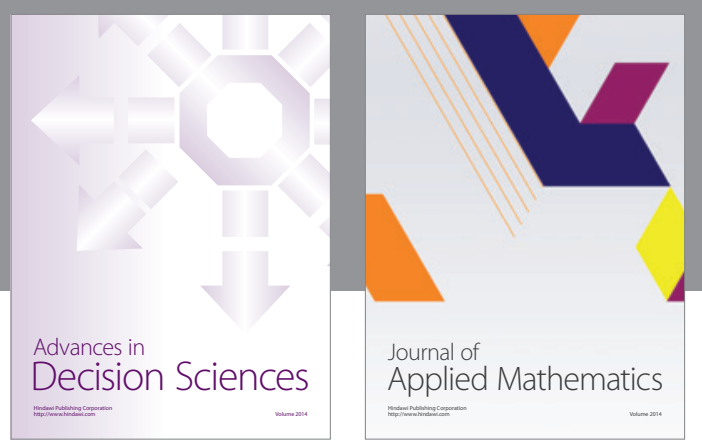

Algebra

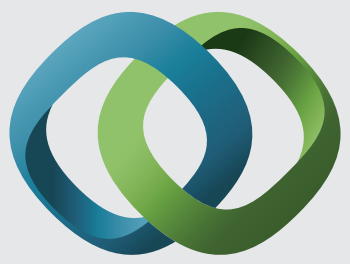

\section{Hindawi}

Submit your manuscripts at

https://www.hindawi.com
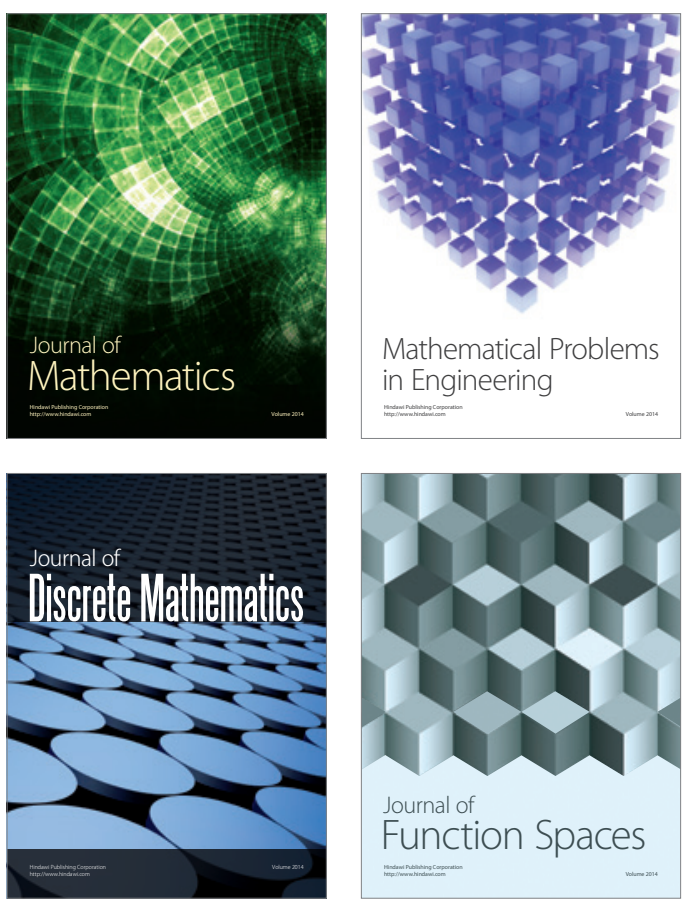

Mathematical Problems in Engineering
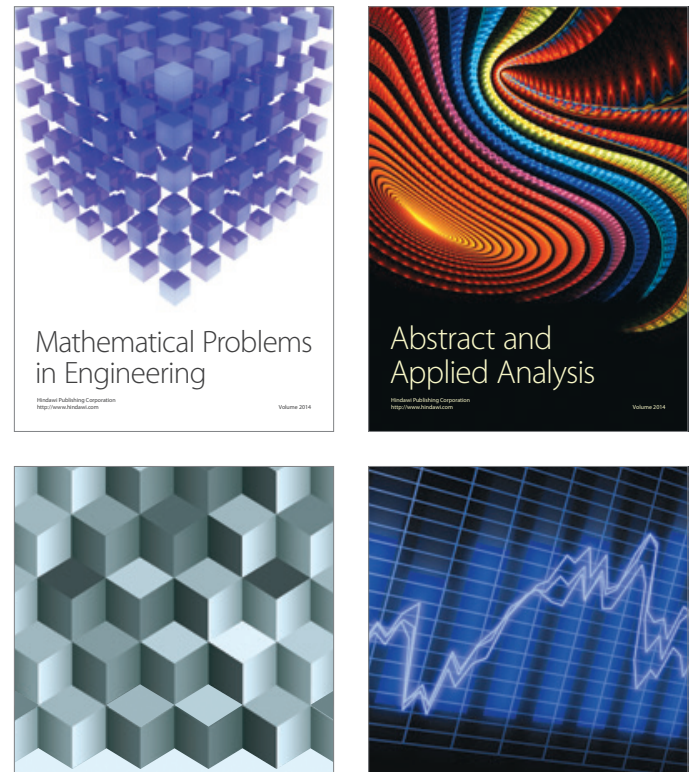

Journal of

Function Spaces

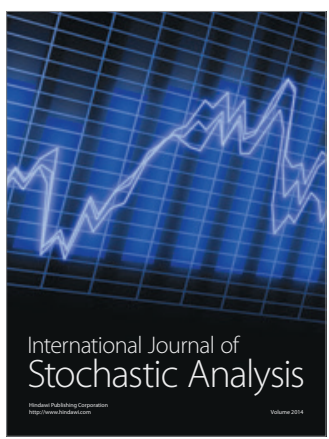

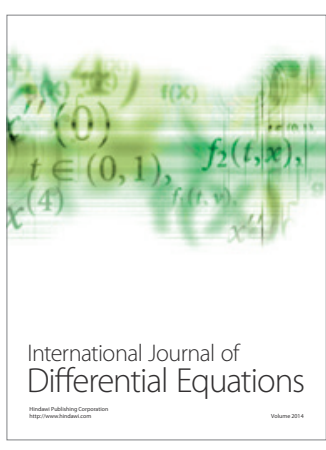
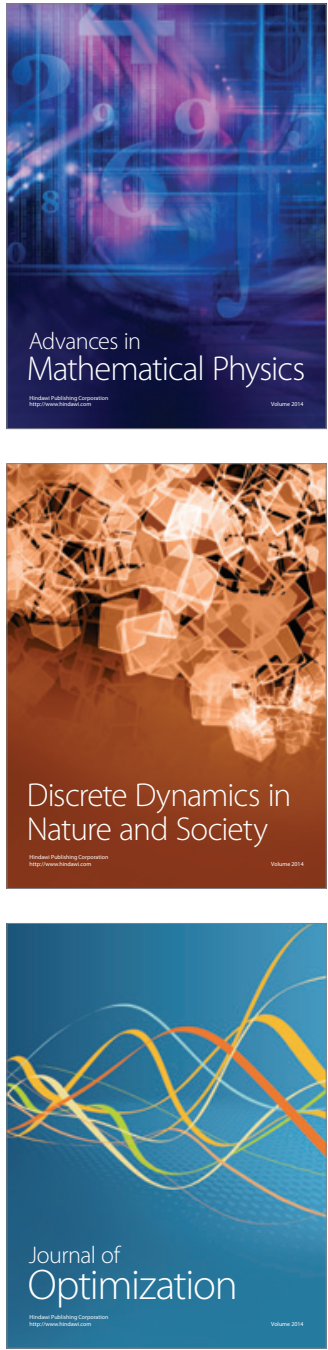